\title{
Calculation of critical points from Helmholtz-energy-explicit mixture models*
}

\author{
Ian H. Bell, Andreas Jäger
}

October 26, 2016

\begin{abstract}
The calculation of the critical points for a mixture of fluids is of practical interest as the calculated critical points can be used to more reliably and efficiently construct phase envelopes. The number of stable critical points found can also provide insight into whether the mixture has an open or closed phase envelope.

In this work we have developed a reliable method for determining all the critical points for a mixture that is modeled with Helmholtz-energy-explicit equations of state. This method extends the algorithms developed in the literature for simpler equations of state to these more complex mixture models. These Helmholtz-energyexplicit equations of state could be either multi-fluid models or transformations of simple cubic equations of state to Helmholtz-energy-explicit forms. This algorithm locks onto the first criticality contour (the spinodal) and traces it to high density, thereby locating all relevant critical points. The necessary analytic derivatives of the residual Helmholtz energy, numerically validated values of the derivatives for validation, sample code, and additional figures and information are provided in the supplemental material.
\end{abstract}

\section{Introduction}

The study of the calculation of the critical points (and critical lines) of mixtures forms a small subset of the broader field of phase equilibria of pure fluids and mixtures. If to begin we restrict ourselves to pure fluids, several phase equilibria are possible - vapor-liquid equilibria, solid-vapor equilibria, solid-solid-vapor triple points, and the like. Mixtures, on the other hand, involve all the complexities of pure fluid phase equilibria, and add another dimension to the problem - the consideration of the mixture composition. The addition of the mixture composition introduces a number of more complex phase equilibria, including critical lines, critical end points, liquid-liquid equilibria, and equilibria with solid phases [1].
The primary goal of this paper is to bring together the work related to the calculation of critical points of mixtures of fluids, and that of the developments in recent years of the high-accuracy Helmholtz-energyexplicit mixture models. Much of the existing literature on the calculation of mixture critical points focuses on well-behaved equations of state (cubics, cubics + excess Gibbs energy, etc.), which simplified the analysis somewhat. In this case we extend the previous models to more complex mixture models, which introduces some numerical challenges, as will be shown below.

The critical line, formed of the critical points, forms an important part of the global phase diagram constituting the vapor pressure curves of the pure components, multi-phase equilibria curves, azeotropic curves, and so on. Global phase diagrams are briefly described in the following section.

\footnotetext{
* Commercial equipment, instruments, or materials are identified only in order to adequately specify certain procedures. In no case does such identification imply recommendation or endorsement by the National Institute of Standards and Technology, nor does it imply that the products identified are necessarily the best available for the purpose. Contribution of the National Institute of Standards and Technology, not subject to copyright in the US
}

(c) 2016. This manuscript version is made available under the Elsevier user license http://www.elsevier.com/open-access/userlicense/1.0/ 


\subsection{Critical Points and Curves}

The majority of the scientific literature on the calculation of critical points of multi-component mixtures dates back to the 1970s. In fact, the history starts much earlier, with the work of Gibbs in 1876, and his establishment of the critical point conditions for a binary mixture[2]:

$$
\left(\frac{\partial^{2} G_{\mathrm{m}}}{\partial x_{1}^{2}}\right)_{T, p}=0 \&\left(\frac{\partial^{3} G_{\mathrm{m}}}{\partial x_{1}^{3}}\right)_{T, p}=0,
$$

where $G_{\mathrm{m}}$ is the molar Gibbs energy, $T$ is the temperature, $p$ is the pressure, and $x_{1}$ is the mole fraction of the first component.

Since 1970, a sizable body of literature has built up related to the calculation of critical points and critical lines for mixtures with two or more components. As a result, the literature survey presented here is intended to give the reader a sense of the more important advances in this field.

Beegle et al. [3] provide a general description of the mathematics involved in assessing the stability of a pure fluid or a mixture at a given state through the use of Legendre transformations applied to the internal energy.

Hicks and Young [4] provide a discussion of the calculation of critical points. They also provide a large body of experimental critical point data collected from their work and curation of other critical point data in literature.

Reid and Beegle[5] subsequently expressed the critical conditions in terms of Legendre transforms, which allows for a straightforward application of the mixture model to calculate the critical point. In this formulation, two matrix determinants (arising from Legendre transforms) must be simultaneously equated with zero, and this is the method further described and implemented in the analysis below.

Gubbins and Twu [6] studied some mixtures based on the combination of model inter-molecular interactions, from which they calculate both phase equilibria and critical loci. They are able to demonstrate a wide range of phase equilibrium behaviors through the use of these simple models.

Heidemann and Khalil [7] applied the criticality conditions and gave practical applications of the calculation of critical points through the use of cubic equations of state. Their work remains one of the most-cited contributions in this field.

Michelsen et al. [8] provided a novel algorithm for calculating critical points, as well as a discussion of the shape of the saturation curves in the vicinity of the critical point. Furthermore, extensive discussions of stability of the critical points and tangent plane distance analyses are provided.
Sadus [9] provided a review of literature on critical point evaluation and a description of some more complex critical point behavior, as well as a discussion of critical point transitions.

Kolár [10] applied the Predictive Soave-RedlichKwong (PSRK) model to several mixtures and calculated critical values with a method similar to that of Heidemann and Khalil [7]. They provide critical loci for a range of mixtures of technical interest.

Michelsen's book [11] provides an overview of the state-of-the-art of calculation of critical points, though as usual, the analysis is focused on cubic equations of state.

In the work of Deiters and Kraska [12], methods are presented to express the derivatives along the critical line, which can then be used to trace the critical line in a computationally efficient manner.

\subsection{Global phase diagrams}

Some of the work described above (e.g., Heidemann and Khalil) emphasized the calculation of the lowest density critical point for a given mixture composition, but there could be additional critical points. Methods that can find all critical points are useful from the standpoint of constructing global phase diagrams.

van Konynenburg and Scott [13] and Scott and van Konynenburg [14] developed much of the fundamental literature on mixture phase type classification. This scheme of classification of mixtures from Type I to Type $\mathrm{V}$ describes the critical curves found in phase diagrams. Type I is the simplest case, a continuous critical line between the critical points of the pure fluids in a binary mixture. More complex mixture classifications have been discovered; see for instance the book of Deiters and Kraska [12], and additional works in the field of global phase diagrams $[15,16]$.

Hicks and Young [17] propose an algorithm for finding the critical points that is similar to the algorithm presented here, both in form and description. Their analysis is focused on cubic equations of state, but here we extend their analysis to multi-fluid models.

Cismondi and Michelsen [18] consider global phase diagrams for mixtures. They investigate a wide range of phase behaviors, and develop an algorithm that can automatically generate the phase diagrams for mixtures from Type I to Type V.

The extension of phase diagrams into three, four, and multi-phase equilibria (including solid phases) has been carried out in the work of Patel [19, 20]. This simulation work involved the calculation of critical points and critical lines, as well as complex multi-phase equilibria, through the use of the homotopy continuation method. 
van Pelt [21] applied the Hicks and Young algorithm [17] to systems modeled through the use of the perturbed hard chain model.

Hoteit et al. [22] propose a new and more reliable algorithm for the calculation of critical points (from the Peng-Robinson equation of state) that is based on nested and bounded iterations of Brent's method. It is compared with the method of Stradi et al. [23] and found to be more than a thousand times faster while they claim similar ability to locate critical points.

\section{$1.3 \quad$ Helmholtz-energy-explicit mixture models}

Cubic equations were the most-common formulation used to model the thermodynamic properties of mixtures prior to the advent of multi-fluid Helmholtzenergy-explicit mixture models, and even now are still in wide use. The majority of the literature dealing with the calculation of critical points described above is thus centered around cubic equation of state models, though much of the analysis can be used for multi-fluid Helmholtz-energy-explicit models as well.

Over the last few decades, multi-fluid Helmholtzenergy-explicit mixture models have been developed, the best known example being the GERG-2004 [24] and GERG-2008 [25] equations of state. These models are based on a framework that had been previously developed in parallel efforts by researchers in Germany and the USA [26, 27, 28].

As there is already a large body of scientific literature on the use of these multi-fluid Helmholtz-energy-explicit mixture models, and the models themselves are quite complex, it is incumbent on those who would extend these models to continue to use the same or functionally similar nomenclature. As much as possible, we have endeavored to ensure consistency with the nomenclature used in the existing literature. Furthermore, it is beyond the scope of this work to provide a complete description of the decades of work that has been carried out in this field. We therefore direct the reader to the relevant literature where possible and provide here a high-level discussion of the multi-fluid Helmholtz-energy-explicit mixture models.

The specific Helmholtz energy $a$ is a fundamental thermodynamic potential; other properties of interest can be obtained through derivatives of the Helmholtz energy. The Helmholtz energy $a$ is expressed as the sum of the ideal-gas contribution $a^{0}$ and the residual contribution $a^{\mathrm{r}}$. In practice the non-dimensionalized Helmholtz energy is used rather than the specific Helmholtz energy, given by

$$
\alpha=\frac{a}{R T}=\alpha^{0}+\alpha^{\mathrm{r}} .
$$

In the analysis considered here, the ideal-gas contribution $\alpha^{0}$ is not required, and will not be discussed further.

The non-dimensionalized residual Helmholtz energy $\alpha^{\mathrm{r}}$ can be obtained as a combination of the equations of state of the pure fluids, through the sum of a linear mixing contribution $\alpha_{\mathrm{LM}}^{\mathrm{r}}$ and a departure contribution $\alpha_{\mathrm{D}}^{\mathrm{r}}$. Algebraically, this yields

$$
\alpha^{\mathrm{r}}(\tau, \delta, \boldsymbol{x})=\alpha_{\mathrm{LM}}^{\mathrm{r}}(\tau, \delta, \boldsymbol{x})+\alpha_{\mathrm{D}}^{\mathrm{r}}(\tau, \delta, \boldsymbol{x}) .
$$

The term $\alpha_{\mathrm{LM}}^{\mathrm{r}}$ is a linear mixing contribution and is given by

$$
\alpha_{\mathrm{LM}}^{\mathrm{r}}(\tau, \delta, \boldsymbol{x})=\sum_{i=1}^{N} x_{i} \alpha_{\mathrm{o} i}^{\mathrm{r}}(\tau, \delta),
$$

where $\alpha_{\mathrm{o} i}^{\mathrm{r}}$ is the residual part of the non-dimensionalized Helmholtz energy of pure component $i$. The contribution $\alpha_{D}^{r}$ expresses the departure from ideal corresponding states behavior:

$$
\alpha_{\mathrm{D}}^{\mathrm{r}}(\tau, \delta, \boldsymbol{x})=\sum_{i=1}^{N-1} \sum_{j=i+1}^{N} x_{i} x_{j} F_{i j} \alpha_{i j}^{\mathrm{r}}(\tau, \delta),
$$

with $\alpha_{i j}^{\mathrm{r}}(\tau, \delta)$ being the binary specific departure functions and $F_{i j}$ being a binary specific factor.

While the departure term of the multi-fluid model is commonly referred to as an "excess" contribution, there is a strong case in the literature [29] against referring to it as the excess contribution and instead referring to it as the residual contribution. Unfortunately, here we already are using the term "residual" to refer to the nonideal contribution, and, due to its prevalence in the existing literature, we therefore refer to $\alpha_{D}^{\mathrm{r}}$ as the departure term.

The independent variables of Eq. 3 are the mole fractions $\boldsymbol{x}$, the reciprocal reduced temperature $\tau$, and the reduced density $\delta . \tau$ and $\delta$ are defined according to:

$$
\delta=\frac{\rho}{\rho_{\mathrm{r}}(\boldsymbol{x})} \text { and } \tau=\frac{T_{\mathrm{r}}(\boldsymbol{x})}{T} .
$$

$T_{\mathrm{r}}(\boldsymbol{x})$ and $\rho_{\mathrm{r}}(\boldsymbol{x})$ are reducing functions for the temperature and density, respectively. These functions solely depend on the composition and not on temperature or density. A more detailed treatment of multi-fluid Helmholtzenergy-explicit mixture models is given, for example, in the publications of GERG 2004 [24] and GERG 2008 [25]. 
While the multi-fluid Helmholtz-energy-explicit mixture model has great flexibility and offers the promise of high accuracy, equations of state that are also of high-accuracy are only available for a relatively small number of fluids (approximately 125 of the most technically important fluids). Furthermore, the complexity of the multi-parameter equations of state often yield nonphysical behavior, as is described in Deiters and Kraska [12], and is demonstrated below. Therefore, it is appealing to use simpler, more well-behaved equations of state to model fluids for which no multi-parameter equation of state exists and/or to replace the entire $\alpha^{\mathrm{r}}$ contribution from Eq. 3.

The work of Bell and Jäger [30] can be used to transform cubic equations of state explicit in pressure (for instance the popular Peng and Robinson [31, 32] and Soave-Redlich-Kwong [33] equations of state) into a form that is explicit in the residual Helmholtz energy $\alpha^{\mathrm{r}}$, with all derivatives with respect to $\tau, \delta$, and composition given by analytic derivatives. This transformation can take one of two forms:

- A one-fluid transformation: the transformed cubic equation of state replaces entirely the multi-fluid $\alpha^{\mathrm{r}}$ from Eq. 3. The binary interaction parameters $k_{i j}$ are taken from tabulated values. The highaccuracy equations of state are not employed.

- One (or more) of the fluids in the multi-fluid model is replaced by a cubic equation of state transformation. The $k_{i j}$ interaction parameters for the equation of state are not considered, and the binary interaction parameters are those of the multi-fluid model.

\section{Critical point derivatives}

As described by Reid and Beegle[5] and Akasaka[34], the criticality conditions defining the location of the critical point can be expressed through the use of Legendre transforms. The advantage of the Legendre transform is that it allows the user to straightforwardly express the critical conditions as determinants of matrices. There are several equivalent sets of independent variables in the transformation, and as demonstrated by Akasaka[34], the use of the amount of substance (number of moles) of the components (as opposed to other more readily handled independent variables) as independent variables yields a more well-scaled set of matrices. This in turn allows for a more reliable solution method.
This section expresses the criticality conditions in a form that can be readily implemented into libraries based on a Helmholtz-energy-explicit mixture modeling framework, notably, NIST REFPROP [35], CoolProp [36], and TREND [37]. The analysis in this section is based on the analysis presented in the GERG model $[24,25]$ and its recent extensions to carbon capture and sequestration applications [38, 39].

\subsection{First and second criticality condi- tions}

In the Legendre-transformed form, the first criticality condition that must be fulfilled at the critical point is $\mathcal{L}_{1}=0$, where $\mathcal{L}_{1}$ can be given by

$$
\mathcal{L}_{1}=\operatorname{det} \mathbf{L}=\left|\begin{array}{ccc}
L_{11} & \ldots & L_{1 N} \\
L_{21} & \ldots & L_{2 N} \\
\vdots & \ddots & \vdots \\
L_{N 1} & \ldots & L_{N N}
\end{array}\right|=0
$$

where the entries in the $\mathbf{L}$ matrix are given by

$$
L_{i j}=\frac{1}{R T}\left(\frac{\partial^{2} A}{\partial n_{i} \partial n_{j}}\right)_{T, V}=\left(\frac{\partial \ln f_{i}}{\partial n_{j}}\right)_{T, V, n_{i}},
$$

where $f_{i}$ is the fugacity of the $i$-th component, as described by Heidemann and Khalil [7]. Here we use the modified matrix as proposed by Michelsen [11]; that is, we divide the entries in the matrix by the product $R T$, which is a valid operation because multiplying (or dividing) a row by a non-zero constant does not impact the equality of the determinant with zero.

The second criticality condition $\mathcal{M}_{1}=0$ can be given by

$$
\mathcal{M}_{1}=\operatorname{det} \mathbf{M}=\left|\begin{array}{ccc}
L_{11} & \ldots & L_{1 N} \\
L_{21} & \ldots & L_{2 N} \\
\vdots & \ddots & \vdots \\
L_{(N-1) 1} & \ldots & L_{(N-1) N} \\
\frac{\partial \mathcal{L}_{1}}{\partial n_{1}} & \ldots & \frac{\partial \mathcal{L}_{1}}{\partial n_{N}}
\end{array}\right|=0
$$

In order to evaluate the determinants in Eqs. 7 and 9, the entries in the matrices must be scaled by the total number of moles, which allows for evaluation of the contributions from Helmholtz-energy-explicit models. The equality of the critical determinants $\mathcal{L}_{1}$ and $\mathcal{M}_{1}$ with zero are not impacted if we multiply a row by a constant, in this case powers of the total number of moles $n$. Thus we can write

$$
\mathcal{L}_{1}^{*}=\operatorname{det} \mathbf{L}^{*}=\left|\begin{array}{ccc}
n L_{11} & \ldots & n L_{1 N} \\
n L_{21} & \ldots & n L_{2 N} \\
\vdots & \ddots & \vdots \\
n L_{N 1} & \ldots & n L_{N N}
\end{array}\right|=0
$$


and

$$
\mathcal{M}_{1}^{*}=\operatorname{det} \mathbf{M}^{*}=\left|\begin{array}{ccc}
n L_{11} & \ldots & n L_{1 N} \\
n L_{21} & \ldots & n L_{2 N} \\
\vdots & \ddots & \vdots \\
n L_{(N-1) 1} & \ldots & n L_{(N-1) N} \\
n^{3} \frac{\partial \mathcal{L}_{1}}{\partial n_{1}} & \ldots & n^{3} \frac{\partial \mathcal{L}_{1}}{\partial n_{N}}
\end{array}\right|=0
$$

where the entries in $\mathcal{L}_{1}^{*}$ are now given by

$$
n L_{i j}=n\left(\frac{\partial \ln f_{i}}{\partial n_{j}}\right)_{T, V, n_{i}},
$$

and where the analytic form of Eq. 12 can be directly evaluated from the mixture model and is given in the supplemental material. The $\mathbf{L}^{*}$ matrix is symmetric because $\left(\frac{\partial \ln f_{i}}{\partial n_{j}}\right)_{T, V}=\left(\frac{\partial \ln f_{j}}{\partial n_{i}}\right)_{T, V}$, which allows for efficient calculation of the entries in the matrix by calculating the upper triangular part of the matrix and mirroring the data into the lower part of the matrix.

In order to obtain the derivatives of the determinant of the unscaled matrix $\mathbf{L}$ with respect to $n_{i}$ that are required to construct the matrix M, Jacobi's formula (see for instance Magnus and Neudecker [40] [section 8.3]) for the derivative of the determinant of a matrix is applied. Jacobi's formula for a matrix $\mathbf{U}$ whose entries are each exclusively a function of the variable $t$ is given by

$$
\frac{\mathrm{d}}{\mathrm{d} t} \operatorname{det} \mathbf{U}(t)=\operatorname{tr}\left(\operatorname{adj}(\mathbf{U}(t)) \frac{\mathrm{d} \mathbf{U}(t)}{\mathrm{d} t}\right) .
$$

The function $\operatorname{tr}()$ is the trace of its argument, and $\operatorname{adj}()$ is the adjugate matrix (the transpose of the cofactor matrix) of its argument. Thus the partial derivatives in the matrix $\mathbf{M}$ are each of the form like

$$
\left(\frac{\partial \mathcal{L}_{1}}{\partial n_{i}}\right)_{T, V, n_{j}}=\operatorname{tr}\left(\operatorname{adj}(\mathbf{L}) \frac{\partial \mathbf{L}}{\partial n_{i}}\right) .
$$

Unfortunately, the $\mathbf{L}$ matrix cannot be directly constructed, and thus we must re-introduce the powers of the amount of substance (number of moles) into Eq. 14 in order to express the equation in terms of $\mathbf{L}^{*}$. Distributing $n^{3}$ into the equation yields

$$
n^{3}\left(\frac{\partial \mathcal{L}_{1}}{\partial n_{i}}\right)_{T, V, n_{j}}=\operatorname{tr}\left([n \cdot \operatorname{adj}(\mathbf{L})] \cdot n^{2} \frac{\partial \mathbf{L}}{\partial n_{i}}\right)
$$

which yields

$$
n^{3}\left(\frac{\partial \mathcal{L}_{1}}{\partial n_{i}}\right)_{T, V, n_{j}}=\operatorname{tr}\left(\operatorname{adj}\left(\mathbf{L}^{*}\right) \cdot n^{2} \frac{\partial \mathbf{L}}{\partial n_{i}}\right)
$$

because $n \cdot \operatorname{adj}(\mathbf{L})=\operatorname{adj}\left(\mathbf{L}^{*}\right)$ resulting from $\mathbf{L}^{*}=n \mathbf{L}$. In general, $k \cdot \operatorname{adj}(\mathbf{A})=\operatorname{adj}(k \cdot \mathbf{A})$ where $k$ is a constant. The derivative term arising from the unscaled matrix $\mathbf{L}$ is given by

$$
n^{2} \frac{\partial \mathbf{L}}{\partial n_{i}}=\left[\begin{array}{ccc}
n^{2} L_{11 i} & \ldots & n^{2} L_{1 N i} \\
n^{2} L_{21 i} & \ldots & n^{2} L_{2 N i} \\
\vdots & \ddots & \vdots \\
n^{2} L_{N 1 i} & \ldots & n^{2} L_{N N i}
\end{array}\right]
$$

and a term of the form $n^{2} L_{i j k}$ is given by

$$
n^{2} L_{i j k}=n^{2}\left(\frac{\partial L_{i j}}{\partial n_{k}}\right)=n^{2} \frac{\partial}{\partial n_{k}}\left(\left(\frac{\partial \ln f_{i}}{\partial n_{j}}\right)_{T, V}\right)_{T, V} .
$$

The analytic form of Eq. 18 is given in the supplemental material. It can be directly evaluated from the mixture model.

\section{$2.2 \tau$ and $\delta$ derivatives of $\mathcal{L}_{1}^{*}$}

For use in later analysis, the derivatives of $\mathbf{L}^{*}$ with respect to each of $\tau$ and $\delta$ are needed. For instance, the first and second partial derivatives of the $\mathbf{L}^{*}$ matrix with respect to $\tau$ are given by

$$
\left(\frac{\partial \mathbf{L}^{*}}{\partial \tau}\right)_{\delta, \bar{x}}=\left[\begin{array}{ccc}
n L_{11 \tau} & \ldots & n L_{1 N \tau} \\
n L_{21 \tau} & \ldots & n L_{2 N \tau} \\
\vdots & \ddots & \vdots \\
n L_{N 1 \tau} & \ldots & n L_{N N \tau}
\end{array}\right]
$$

and

$$
\left(\frac{\partial^{2} \mathbf{L}^{*}}{\partial \tau^{2}}\right)_{\delta, \bar{x}}=\left[\begin{array}{ccc}
n L_{11 \tau \tau} & \ldots & n L_{1 N \tau \tau} \\
n L_{21 \tau \tau} & \ldots & n L_{2 N \tau \tau} \\
\vdots & \ddots & \vdots \\
n L_{N 1 \tau \tau} & \ldots & n L_{N N \tau \tau}
\end{array}\right]
$$

where an entry in the first $\tau$ derivative matrix is of the form

$$
n L_{i j \tau}=\frac{\partial}{\partial \tau}\left(n \frac{\partial \ln f_{i}}{\partial n_{j}}\right)_{\delta, \bar{x}}
$$

and an entry in the second $\tau$ derivative matrix is of the form

$$
n L_{i j \tau \tau}=\frac{\partial^{2}}{\partial \tau^{2}}\left(n \frac{\partial \ln f_{i}}{\partial n_{j}}\right)_{\delta, \bar{x}} .
$$

The analytic forms of these derivatives are given in the supplemental material.

Similarly, for the partial derivatives with respect to $\delta$, entries in the $\delta$ derivative matrices can be given in the form

$$
\begin{aligned}
n L_{i j \delta} & =\frac{\partial}{\partial \delta}\left(n \frac{\partial \ln f_{i}}{\partial n_{j}}\right)_{\tau, x} \\
n L_{i j \delta \delta} & =\frac{\partial^{2}}{\partial \delta^{2}}\left(n \frac{\partial \ln f_{i}}{\partial n_{j}}\right)_{\tau, x},
\end{aligned}
$$

where, again, the form of these derivatives can be found in the supplemental material. 


\section{Algorithm for finding critical points}

For a given mixture composition, the first and second criticality conditions $\mathcal{L}_{1}^{*}=0$ and $\mathcal{M}_{1}^{*}=0$ can each be expressed as functions of two state variables. In this case, the most readily understandable set of state variables would be temperature and volume (or density), though the mixture model is based on the use of $\tau$ and $\delta$ as independent variables. To limit the amount of additional work required, $\tau$ and $\delta$ are selected as the independent variables to be determined, and a post-processing step can be used to obtain the temperature and density.

The goal of the critical point solver is to iterate on $\tau$ and $\delta$ to find the pair of values that enforce $\mathcal{L}_{1}^{*}=$ $\mathcal{M}_{1}^{*}=0$. To that end, a classical Newton-Raphson iteration scheme is employed, leveraging the fact that the Jacobian matrix can be constructed analytically.

The challenge of the application of the NewtonRaphson method is that quite good estimates of the critical point are required, as noted by several authors in the literature $[7,11,12]$. Additionally, the mixture model can predict numerous values where the criticality conditions $\mathcal{L}_{1}^{*}=\mathcal{M}_{1}^{*}=0$ are met, some of which correspond to stable critical points, others which correspond to unstable critical points, and yet others that are exclusively numerical artifacts and have no physical significance.

In this section, a description of the Newton-Raphson solver is presented (when a reasonably good guess value is already known for the critical point). Additionally, a method is presented that can find all the critical points for a mixture by first finding a point along the $\mathcal{L}_{1}^{*}=0$ contour (the spinodal) at a low density and then tracing this contour to very high density, thereby finding all the relevant critical points along the $\mathcal{L}_{1}^{*}=0$ contour.

\subsection{Critical point solver}

The Newton-Raphson system for the two-dimensional solver in $\tau$ and $\delta$ for the criticality conditions is given by

$$
\mathbf{J} \Delta \boldsymbol{X}=-\left[\begin{array}{c}
\mathcal{L}_{1}^{*}(\tau, \delta) \\
\mathcal{M}_{1}^{*}(\tau, \delta)
\end{array}\right]
$$

where $\boldsymbol{X}$ is given by $\boldsymbol{X}=\left[\begin{array}{ll}\tau & \delta\end{array}\right]^{T}$ and the step $\Delta \boldsymbol{X}$ is given by $\Delta \boldsymbol{X}=\left[\begin{array}{ll}\Delta \tau & \Delta \delta\end{array}\right]^{T}$. The Jacobian is given by

$$
\mathbf{J}=\left[\begin{array}{cc}
\left(\frac{\partial \mathcal{L}_{1}^{*}}{\partial \tau}\right)_{\delta} & \left(\frac{\partial \mathcal{L}_{1}^{*}}{\partial \delta}\right)_{\tau} \\
\left(\frac{\partial \mathcal{M}_{1}^{*}}{\partial \tau}\right)_{\delta} & \left(\frac{\partial \mathcal{M}_{1}^{*}}{\partial \delta}\right)_{\tau}
\end{array}\right] .
$$

The Newton-Raphson algorithm operates by solving Eq. 25 for $\Delta \boldsymbol{X}$, and updating $\boldsymbol{X}$ with

$$
\boldsymbol{X}_{i+1}=\boldsymbol{X}_{i}+\Delta \boldsymbol{X}
$$

Accurate initial values for $\boldsymbol{X}_{0}$ are required to yield a good starting point for the method.

Jacobi's formula (see Eq. 13) is again applied in order to evaluate the partial derivative terms in the Jacobian matrix. The derivative terms in Eq. 26 are expanded in terms of the matrices $\mathbf{L}^{*}$ and $\mathbf{M}^{*}$ (upon which the determinants are based). Thus the Jacobian can be given by

$$
\mathbf{J}=\left[\begin{array}{cc}
\operatorname{tr}\left(\operatorname{adj}\left(\mathbf{L}^{*}\right) \frac{\partial \mathbf{L}^{*}}{\partial \tau}\right) & \operatorname{tr}\left(\operatorname{adj}\left(\mathbf{L}^{*}\right) \frac{\partial \mathbf{L}^{*}}{\partial \delta}\right) \\
\operatorname{tr}\left(\operatorname{adj}\left(\mathbf{M}^{*}\right) \frac{\partial \mathbf{M}^{*}}{\partial \tau}\right) & \operatorname{tr}\left(\operatorname{adj}\left(\mathbf{M}^{*}\right) \frac{\partial \mathbf{M}^{*}}{\partial \delta}\right)
\end{array}\right] .
$$

The derivatives of entries in the $\mathbf{L}^{*}$ matrix are given by equations 21 and 23; the analytic forms of these equations can be found in the supplemental material.

For $\mathbf{M}^{*}$, the first $\mathrm{N}-1$ rows of the $\partial \mathbf{M}^{*} / \partial \tau$ matrix are exactly the same as those from the $\partial \mathbf{L}^{*} / \partial \tau$ matrix. The last row in the $\partial \mathbf{M}^{*} / \partial \tau$ matrix involves terms of the form

$$
\frac{\partial}{\partial \tau}\left(\mathbf{M}_{N i}^{*}\right)=\frac{\partial}{\partial \tau}\left[\operatorname{tr}\left(\operatorname{adj}\left(\mathbf{L}^{*}\right) \cdot\left(n^{2} \frac{\partial \mathbf{L}}{\partial n_{i}}\right)\right)\right] .
$$

Trace and derivative operations are commutative, so this derivative can be expressed (after applying the product rule for matrices) as

$$
\frac{\partial}{\partial \tau}\left(\mathbf{M}_{N i}^{*}\right)=\operatorname{tr}\left[\begin{array}{c}
\left(n^{2} \frac{\partial \mathbf{L}}{\partial n_{i}}\right) \cdot \frac{\partial}{\partial \tau}\left[\operatorname{adj}\left(\mathbf{L}^{*}\right)\right] \\
+\operatorname{adj}\left(\mathbf{L}^{*}\right) \frac{\partial}{\partial \tau}\left[n^{2} \frac{\partial \mathbf{L}}{\partial n_{i}}\right]
\end{array}\right] .
$$

The derivative term arising from the $n^{2} \cdot \partial \mathbf{L} / \partial n_{i}$ term is given by

$$
\frac{\partial}{\partial \tau}\left(n^{2} \frac{\partial \mathbf{L}}{\partial n_{i}}\right)=\left[\begin{array}{ccc}
n^{2} L_{11 i \tau} & \ldots & n^{2} L_{1 N i \tau} \\
n^{2} L_{21 i \tau} & \ldots & n^{2} L_{2 N i \tau} \\
\vdots & \ddots & \vdots \\
n^{2} L_{N 1 i \tau} & \ldots & n^{2} L_{N N i \tau}
\end{array}\right],
$$

which involves terms of the form

$$
n^{2} L_{i j k \tau}=\frac{\partial}{\partial \tau}\left(n^{2} L_{i j k}\right),
$$

with $n^{2} L_{i j k}$ from Eq. 18. The derivative in Eq. 32 can be found in the supplemental material. A similar set of derivatives is obtained for the partial derivatives taken with respect to $\delta$, whose forms are also in the supplemental material.

The final derivative term $\partial\left[\operatorname{adj}\left(\mathbf{L}^{*}\right)\right] / \partial \tau$ requires further discussion. The adjugate of a matrix $\mathbf{A}$ (with entries that are exclusively a function of $t$ ) is given by the operator $\mathbf{A}^{\mathrm{A}}=\operatorname{adj}(\mathbf{A})$, and has elements given by

$$
\mathbf{A}_{i j}^{\mathrm{A}}=(-1)^{i+j} M_{j i},
$$


where $M_{j i}$ is the $j i$ minor of the matrix $\mathbf{A}$.

The minor $M_{j i}$ of a matrix $\mathbf{A}$ is obtained by taking the determinant of the sub-matrix $A_{j i}^{\prime}$ formed by removing row $j$ and column $i$ from A. This can be expressed as

$$
M_{j i}=\operatorname{det}\left(A_{j i}^{\prime}\right) .
$$

Thus, in general, the derivative of an entry in the adjugate matrix is given by

$$
\frac{\mathrm{d}}{\mathrm{d} t}\left(\mathbf{A}_{i j}^{\mathrm{A}}\right)=\frac{\mathrm{d}}{\mathrm{d} t}\left[(-1)^{i+j} \operatorname{det}\left(A_{j i}^{\prime}\right)\right],
$$

which can be expanded through the use of Jacobi's formula one final time to read

$$
\frac{\mathrm{d}}{\mathrm{d} t}\left(\mathbf{A}_{i j}^{\mathrm{A}}\right)=(-1)^{i+j} \operatorname{tr}\left(\operatorname{adj}\left(A_{j i}^{\prime}\right) \frac{\mathrm{d} A_{j i}^{\prime}}{\mathrm{d} t}\right) .
$$

The reader should be advised that the adjugate of a $1 \times 1$ matrix is equal to 1.

\subsection{Critical point finder}

While some authors have proposed methods to robustly find all the critical points [23, 17, 12], a subset of that analysis will be developed here. In practice, the critical points that are of interest are those that correspond to positive pressures, are stable, and lie along the highesttemperature branch of the $\mathcal{L}_{1}^{*}=0$ contour.

As an introduction, we present the criticality contours from a well-behaved mixture model in order to explain some of the more important features. Figure 1 demonstrates the critical contours for an equimolar $n$ hexane $+n$-heptane mixture as calculated by the multifluid model, with the mixture parameters and pure fluid parameters as given in Table 2. A few other curves are also plotted in order to provide context for the critical contours. The phase envelope is shown in order to confirm that the calculated critical point is co-incident with the phase envelope. Furthermore, the "ghost" critical points (numerical artifacts described further in section 6.1) are entirely enclosed within the phase envelope (towards higher $\tau$, or lower temperature). Critical points must lie along a phase envelope because the phase envelope is the locus of points that satisfies vapor-liquid equilibrium (equality of component fugacities, material balance, etc.).

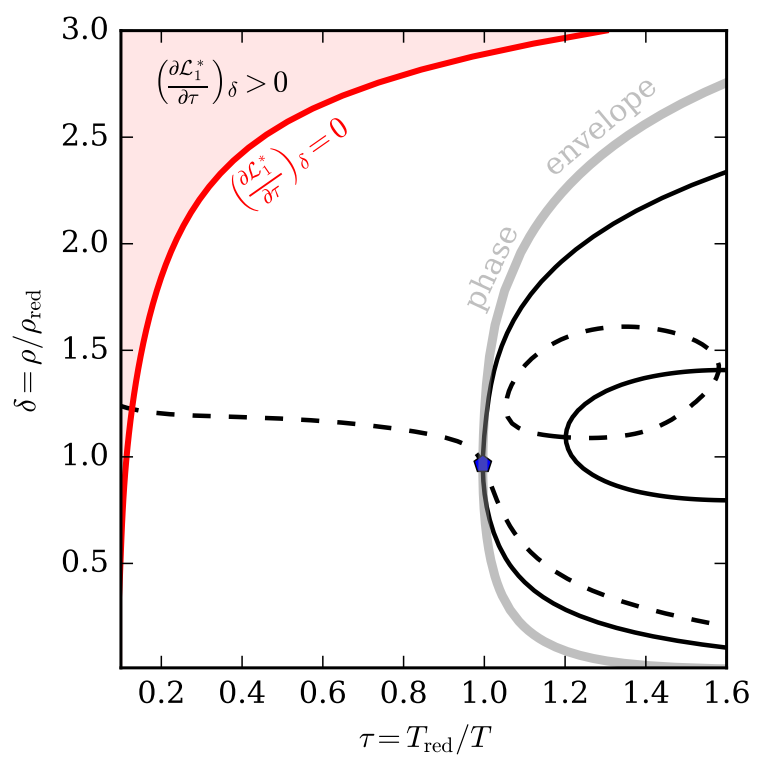

Figure 1: Criticality contours (solid: $\mathcal{L}_{1}^{*}=0$, dashed: $\mathcal{M}_{1}^{*}=0$ ) of an equimolar $n$-hexane $+n$-heptane mixture from the multi-fluid model, with the model parameters given by Table 2 . The critical point is identified with a marker.

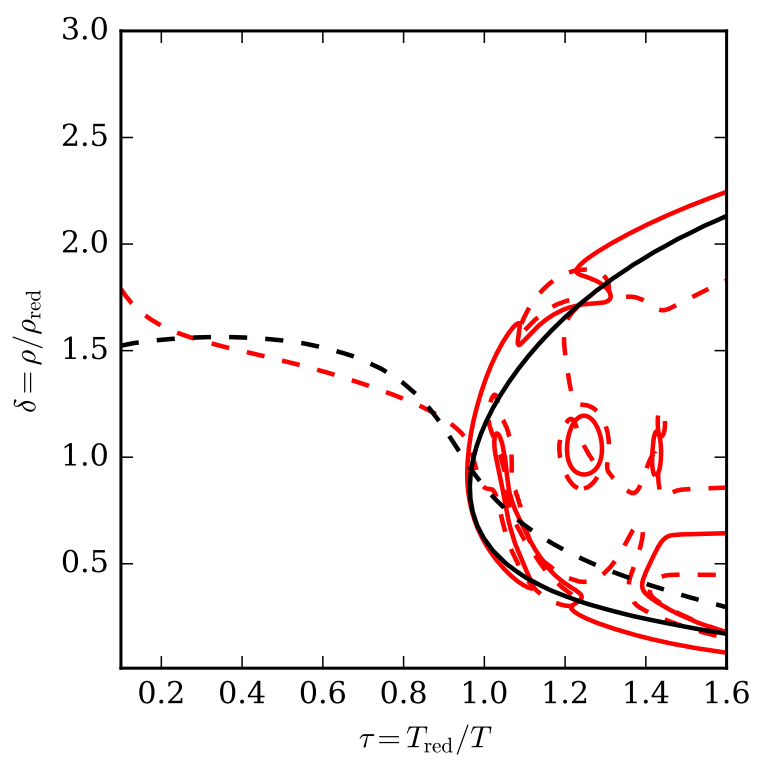

Figure 2: Criticality contours (solid: $\mathcal{L}_{1}^{*}=0$, dashed: $\mathcal{M}_{1}^{*}=0$ ) of an equimolar nitrogen + methane mixture (red: multi-fluid model, black: SRK transformations of pure fluids in multi-fluid model), with the model parameters from Table 2 . 
As a counter-example of a poorly behaved mixture model, we overlay the criticality contours for an equimolar mixture of nitrogen and methane in Figure 2 as calculated with SRK transformations of the pure fluids in a multi-fluid model, and the multi-fluid model itself. The mixture parameters and pure fluid parameters are as given in Table 2. This mixture demonstrates challenging behavior; when the multi-fluid model is employed, the $\mathcal{L}_{1}^{*}=0$ contour is not smooth, which causes significant challenges for the critical point finding routine. On the other hand, when SRK is used, either as a one-fluid model or in pure-fluid transformations in the multi-fluid model, the critical contours are much more smooth. Figure 2 demonstrates that criticality contour plots serve as a robust check of the physically-reasonable behavior of the pure-fluid equations of state when employed in multifluid mixture models.

\subsubsection{Maximum temperature contour}

To begin the critical point finding routine along the maximum temperature $\mathcal{L}_{1}^{*}=0$ contour, the value of $\tau$ corresponding to $\mathcal{L}_{1}^{*}=0$ is found at a low density value. This value of density should be selected such that all critical points (if they exist), are above this density value. In this work, we have found that selecting a density value of $0.5 \cdot \rho_{\text {red }}(\boldsymbol{x})$ was a good choice for a low-density starting value. A first guess for the temperature value is selected that should be well above the temperature corresponding to the highest temperature $\mathcal{L}_{1}^{*}=0$ contour, where we found that $1.5 \cdot T_{\text {red }}(\boldsymbol{x})$ was a good choice. In the case that the derivative $\partial\left(\mathcal{L}_{1}^{*}\right) / \partial \tau$ is positive, the starting value of $\tau$ is increased so that the proper solution is found. Figure 1 shows the contour where the derivative $\partial\left(\mathcal{L}_{1}^{*}\right) / \partial \tau$ is zero, and points towards higher values of $\tau$ are valid starting points. In practice, the user must be careful to select a temperature value that is not too high ( $\tau$ value that is not too low), otherwise the solver might tend to overshoot and find a non-physical critical point inside the spinodal. One means of more reliably finding the lowest $\tau$ value (highest temperature) satisfying $\mathcal{L}_{1}^{*}=0$ for a low density value is to use a higher-order derivative-based iterative solver. It was found by experience that a first-order derivative-based iterative solver (Newton's method) required a reasonably accurate guess value for the temperature, while the use of a secondorder derivative-based iterative solver (Halley's method) was far less sensitive to the initial guess for $\tau$, allowing a higher temperature guess value while also avoiding the problems of overshoot and the selection of a lowertemperature root.
In Halley's method, the first and second derivatives of the residual function with respect to the independent variable are required. The development of the derivatives required for the Jacobian above describes the first partial derivative of $\mathcal{L}_{1}^{*}$ with respect to $\tau$ (Eqs. 26 and 28 ), and the second partial derivative of $\mathcal{L}_{1}^{*}$ with respect to $\tau$ can be given by

$$
\left(\frac{\partial^{2} \mathcal{L}_{1}^{*}}{\partial \tau^{2}}\right)_{\delta}=\operatorname{tr}\left[\begin{array}{c}
\left(\frac{\partial \mathbf{L}^{*}}{\partial \tau}\right)_{\delta, x} \frac{\partial}{\partial \tau}\left[\operatorname{adj}\left(\mathbf{L}^{*}\right)\right] \\
+\operatorname{adj}\left(\mathbf{L}^{*}\right)\left(\frac{\partial^{2} \mathbf{L}^{*}}{\partial \tau^{2}}\right)_{\delta, \boldsymbol{x}}
\end{array}\right]
$$

The next guess for $\tau$ can then be found by the iterative updating step

$$
\tau_{\text {new }}=\tau_{\text {old }}-\frac{2 \mathcal{L}_{1}^{*}\left(\frac{\partial \mathcal{L}_{1}^{*}}{\partial \tau}\right)_{\delta}}{2\left[\left(\frac{\partial \mathcal{L}_{1}^{*}}{\partial \tau}\right)_{\delta}\right]^{2}-\mathcal{L}_{1}^{*}\left(\frac{\partial^{2} \mathcal{L}_{1}^{*}}{\partial \tau^{2}}\right)_{\delta}}
$$

Eq. 38 is repetitively applied until the value of $\left|\mathcal{L}_{1}^{*}\right|$ is sufficiently close to zero. This therefore defines the first point along the $\mathcal{L}_{1}^{*}=0$ contour.

\subsection{2 $\mathcal{L}_{1}^{*}=0$ contour tracer}

After the initial point along the $\mathcal{L}_{1}^{*}=0$ contour has been found, the solver switches into a new mode where it traces along the $\mathcal{L}_{1}^{*}=0$ contour. Along this contour, if critical points exist, they are points that satisfy $\mathcal{L}_{1}^{*}=\mathcal{M}_{1}^{*}=0$. In order to trace along the contour, an angle variable $\theta$ is introduced that yields a vector pointing along the contour (in the direction of increasing density). At the current value of $\left(\tau_{k}, \delta_{k}\right)$, the newly predicted point in $\tau$ - $\delta$ space is defined by

$$
\begin{aligned}
& \tau_{k+1}=\tau_{k}+R_{\tau} \cos (\theta) \\
& \delta_{k+1}=\delta_{k}+R_{\delta} \sin (\theta)
\end{aligned}
$$

The value for $\theta$ that yields $\mathcal{L}_{1}^{*}=0$ is iteratively calculated. In the first step, $\theta$ is searched in the domain $(0, \pi)$ through the use of a bounded solver - here Brent's method. Once the first value for $\theta$ is obtained, the next step begins with the guess value that $\theta$ equals its previous value. The $\mathcal{L}_{1}^{*}=0$ contour is in general smooth (see Fig. 1, or as a counter-example, Fig. 2), therefore the values of $\theta$ also change slowly. After the first step, derivative-based methods can be used to obtain $\theta$. In particular, Newton's method is used to iteratively update $\theta$; the derivative of $\mathcal{L}_{1}^{*}$ with respect to the angle $\theta$ is required, which can be given by

$$
\frac{\partial \mathcal{L}_{1}^{*}}{\partial \theta}=\left(\frac{\partial \mathcal{L}_{1}^{*}}{\partial \tau}\right)_{\delta} \frac{\mathrm{d} \tau}{\mathrm{d} \theta}+\left(\frac{\partial \mathcal{L}_{1}^{*}}{\partial \delta}\right)_{\tau} \frac{\mathrm{d} \delta}{\mathrm{d} \theta}
$$


where

$$
\frac{\mathrm{d} \tau}{\mathrm{d} \theta}=-R_{\tau} \sin \theta
$$

and

$$
\frac{\mathrm{d} \delta}{\mathrm{d} \theta}=R_{\delta} \cos \theta
$$

and where $\theta$ can be updated by repetitive applications of

$$
\theta_{\text {new }}=\theta_{\text {old }}-\frac{\mathcal{L}_{1}^{*}}{\left[\frac{\partial \mathcal{L}_{1}^{*}}{\partial \theta}\right]} .
$$

In practice, since the curvature of the $\mathcal{L}_{1}^{*}=0$ contour is quite gentle for well-behaved mixture models, only one or two updates of the value of $\theta$ are needed, making for a computationally efficient method of tracing the contour.

In spite of the generally reliable and robust behavior of this tracing methodology, there are times when the contour tracer would prefer to change direction and trace back towards lower $\delta$ values. In order to avoid this, the obtained value of $\theta$ and the previous value of $\delta$ are compared. If their values differ by more than $\pi / 2$ radians ${ }^{1}$, the tracer has made a sharp change in direction. A bounded solver is then used (with a domain centered around the previous value of $\theta$ ) in order to ensure that the tracer continues in the same direction.

The values of the radii $R_{\tau}$ and $R_{\delta}$ were selected by manual iteration to be a compromise between speed (fewer steps are required for larger radii) and reliability (less likely to hit a "ghost" contour for smaller radii). For the multi-fluid model, the values of $R_{\tau}=0.1$ and $R_{\delta}=0.025$ were ultimately selected. Due to the generally more well behaved shapes of the criticality contours when using one-fluid SRK model is used (as well as when SRK transformations in the multi-fluid model are used), the search radii could be much larger. For the SRK models, multiplying the radii for the multi-fluid model by a factor of approximately 5 (after also applying the scaling of section 4.1), proved to be a fair compromise of speed and reliability.

In the process of tracing the $\mathcal{L}_{1}^{*}=0$ contour, the value of $\mathcal{M}_{1}^{*}$ is calculated at each step. If the $\operatorname{sign}$ of $\mathcal{M}_{1}^{*}$ changes between the values of $\left(\tau_{k}, \delta_{k}\right)$ and $\left(\tau_{k+1}, \delta_{k+1}\right)$, these values for $\tau$ and $\delta$ must necessarily bound a critical point. A call is then made to the Newton-Raphson critical point calculation routine from section 3.1, which then efficiently refines the solution for the critical point because the guess value for the critical temperature and density is nearly co-incident with the true critical point.
Stopping condition The tracer follows the spinodal from lower densities towards higher densities, locating all the critical points that it can, until it reaches the stopping condition, detailed in this section.

For the one-fluid SRK mixture model, there is a minimum specific volume that is physically allowed - the covolume $b_{m}$. The one-fluid SRK models begin to show some undesirable behavior in approaching the covolume, and for the one-fluid models, the stopping condition is that the volume must be greater than $1.1 b_{\mathrm{m}}$. Stradi et al. [23] used the same bound of $1.1 b_{\mathrm{m}}$ in their work. If this condition is not fulfilled, the tracer terminates. There may be some critical points between $1.1 b_{\mathrm{m}}$ and $b_{\mathrm{m}}$, but they are mostly critical points that are of less interest, at negative pressures, unstable, or all of the above.

For the multi-fluid models, the stopping condition is somewhat more difficult to define. While the one-fluid SRK model has a physical bound on the allowed volume, the multi-fluid model is entirely empirical in nature, and less constrained by physical behavior. As such, the stopping conditions for the multi-fluid model (any of which terminate the tracer) are:

- The pressure exceeds $500 \mathrm{MPa}$.

- The reduced density $\delta$ exceeds 5 .

- The reciprocal reduced temperature $\tau$ exceeds 5 .

- The reduced density $\delta$ exceeds 1.5 and the tracer has a sharp change in its direction (other than a Uturn). This can be caused by problems in the purefluid equation of state at low temperature (high $\tau$ ). As an example, see Fig. 2.

\subsection{Stability of critical point}

It is necessary to implement a full tangent plane distance analysis to evaluate whether a critical point is stable or not. The tangent plane distance analysis is a necessary and sufficient condition that can guarantee that the critical point is stable[11]. Unfortunately, this guarantee comes at a price. In principle, the tangent plane analysis requires a global search over the entire test composition domain, but we simplify the stability search by evaluating two trial compositions for heavy and light test phases.

\footnotetext{
${ }^{1}$ The true angle difference function should be used in order to properly handle the fact that $\theta$ is $2 \pi$ periodic. The difference between the angles of 0.01 radians and $2 \pi-0.01$ radians should be 0.02 radians, not $2 \pi-0.02$ radians.
} 
In this work we applied the algorithm of Gernert et al. [38] as applied to the multi-fluid Helmholtz-energyexplicit mixture model. The algorithm is based on the work of Michelsen [41, 42] and was developed with an emphasis on multi-fluid Helmholtz models. These models pose significantly more challenging numerical behavior than less complex equations of state (like cubic equations of state), which are often used for critical point and phase equilibrium calculations. The applicability and reliability of this method will be discussed in section 5 .

\section{Implementation}

$\mathrm{A} \mathrm{C}++$ implementation of the algorithm for identifying critical points and evaluating their stability is provided in the supplemental material; information is available in the supplemental material about how to compile the $\mathrm{C}++$ sources. Following publication of this work, it is intended to implement this algorithm in the standard reference NIST REFPROP library, where the critical point calculation routines will be used in order to more efficiently construct phase envelopes for mixtures of fixed composition.

\subsection{Considerations for one-fluid cubic equations of state}

In the generalized transformation of pressure-explicit cubic equations of state to Helmholtz energy equations of state of Bell and Jäger [30], the reducing parameters $T_{\text {red }}$ and $\rho_{\text {red }}$ are positive numerical constants (not a function of composition). Unlike the multi-fluid model, in the one-fluid model there is no link between the critical densities and/or temperatures of the pure components and the reducing parameters. In the case that a calculation begins with a pair of $T, \rho$, a round-trip evaluation of $T, \rho \rightarrow \tau, \delta \rightarrow T, \rho$ poses no numerical problems as the (constant) reducing parameters cancel. On the other hand, when a calculation begins with a given value of $(\tau, \delta)$, the differences between the multi-fluid and onefluid models become evident.
As an example, when determining the first point along $\mathcal{L}_{1}^{*}=0$ at low $\delta$, as described above we begin our one-dimensional search at a value of $\tau$ of approximately $2 / 3$ and a value of $\delta$ of approximately $1 / 2$. These values have a physical significance in the multi-fluid model. For the one-fluid model, due to the arbitrary nature of the reducing parameters, it is necessary to rescale $\tau$ and $\delta$ to put them on a more physical footing. In order to do this, we assume a linear mixing of the critical volume and critical temperature in the one-fluid model, and use these linear mixing pseudo-reducing parameters in order to yield similar values of $T$ and $\rho$. Mathematically, this is expressed as:

$$
\begin{gathered}
\delta_{\mathrm{OF}}=\delta_{\mathrm{MF}} \frac{1}{\rho_{\mathrm{r}, \mathrm{OF}}\left(\sum_{i} x_{i} v_{\mathrm{c}, i}\right)} \\
\tau_{\mathrm{OF}}=\tau_{\mathrm{MF}} \frac{T_{\mathrm{r}, \mathrm{OF}}}{\left(\sum_{i} x_{i} T_{\mathrm{c}, i}\right)}
\end{gathered}
$$

where the subscript OF pertains to the one-fluid model, and MF to the multi-fluid model. It is generally the case that the critical volume $v_{\mathrm{c}, i}$ is unknown for cubic equations of state. Therefore, $v_{\mathrm{c}, i}$ must be estimated, either by applying the volume translation method of Péneloux [43] to critical volumes estimated based on the critical compressibility factor of the equation of state (a constant), or with the use of an approximate formulation, such as

$$
v_{\mathrm{c}, i} \approx 2.141\left(\frac{T_{\mathrm{c}, i}}{p_{\mathrm{c}, i}}\right)+7.773 \times 10^{-6}
$$

where $v_{\mathrm{c}, i}$ is in $\mathrm{m}^{3} \cdot \mathrm{mol}^{-1}$ and $T_{\mathrm{c}, i} / p_{\mathrm{c}, i}$ is in $\mathrm{K} \cdot \mathrm{Pa}^{-1}$. This equation was obtained by fitting the critical parameters for all the pure fluids with multi-parameter equations of state. This equation predicts $94 \%$ of the critical volumes within $10 \%$, which is adequate for purposes of scaling the values for $\delta$. 


\section{Validation of the implementa- tion}

In order to demonstrate that our implementations of the critical point routines as well as the stability calculations in the thermophysical property libraries CoolProp and TREND are correct, a comparison with the work of Stradi et al. [23] is given in Table 1. In this table, calculated critical points for different compositions of the binary mixture methane $\left(\mathrm{CH}_{4}\right)+$ hydrogen sulfide $\left(\mathrm{H}_{2} \mathrm{~S}\right)$ are given. The critical points indicated "Stradi et al. - SRK" are results of Stradi et al. [23], which were taken from their article and reprinted for comparison. The results indicated "This work - SRK" are the results for critical points in the binary mixture $\mathrm{CH}_{4}+\mathrm{H}_{2} \mathrm{~S}$ calculated with the implementation of the SRK in TREND [37].

In order to get comparable results, values of constants of the SRK equation were adjusted to match the values Stradi et al. [23] used. Unfortunately, Stradi et al. did not provide the value that they used for the gas constant $R$. Hence, we assumed that they used a value of $R=8.31451 \mathrm{~J} \cdot \mathrm{mol}^{-1} \cdot \mathrm{K}^{-1}$, which was the standard value at the time when they did their study. As in Stradi et al. [23], the values for the critical parameters and acentric factors of methane and hydrogen sulfide have been taken from Reid et al. [44]; they are: $T_{\mathrm{c}, \mathrm{CH}_{4}}=$ $190.4 \mathrm{~K}, p_{\mathrm{c}, \mathrm{CH}_{4}}=4.6 \mathrm{MPa}, \omega_{\mathrm{CH}_{4}}=0.011, T_{\mathrm{c}, \mathrm{H}_{2} \mathrm{~S}}=$ $373.2 \mathrm{~K}, p_{\mathrm{c}, \mathrm{H}_{2} \mathrm{~S}}=8.94 \mathrm{MPa}$, and $\omega_{\mathrm{H}_{2} \mathrm{~S}}=0.097$.

Table 1 shows that the calculated critical points and their stability are in very good agreement with the results of Stradi et al. If at certain overall compositions no values for the critical temperature, density, or pressure is given, then according to the equation of state no critical points exist for this overall composition, or they were not found. With the algorithm presented in this paper, some critical points at negative pressures for methanerich mixtures have been found, which were not given by Stradi et al. The algorithm that was used for testing the stability [38] is TP-based and thus the stability of points at negative pressures could not be tested, which is indicated with "NA" in Table 1. The entries "-" in Table 1 indicate critical points that were not able to be calculated with the respective model. Furthermore, the possible formation of solid phases was not tested either, since no equation of state for the solid phases of $\mathrm{CH}_{4}$ and $\mathrm{H}_{2} \mathrm{~S}$ was employed in this study.
The critical points in Table 1 indicated "This work - Helmholtz" are the predicted critical points from the multi-fluid Helmholtz energy equation of state for methane [45] in combination with the multiparameter Helmholtz energy equation of state for hydrogen sulfide [46] and mixing rules from GERG-2008 [25] ( $\beta_{T}=$ $1.011090031, \gamma_{T}=0.961155729, \beta_{v}=1.012599087$, and $\gamma_{v}=1.040161207$, with no departure function). Calculated critical temperatures, molar volumes, and pressures of the mixtures differ to some extent from the SRK results but are in good agreement, especially the temperatures and pressures, and less-so the volumes. However, some of the critical points that the one-fluid SRK model predicts to be unstable are stable according to the multi-fluid model. Furthermore, the multi-fluid model predicts the existence of critical points that are not predicted by the one-fluid model. This behavior, however, is not unique to the multi-fluid Helmholtz-energy-explicit models but it was found that the existence and also the stability of critical points is very sensitive to the model parameters for the binary mixture $\mathrm{CH}_{4}+\mathrm{H}_{2} \mathrm{~S}$. For example, when changing the critical parameters and acentric factors of methane and hydrogen sulfide in the SRK to the values given by Poling et al. [47] $\left(T_{\mathrm{c}, \mathrm{CH}_{4}}=\right.$ $190.56 \mathrm{~K}, p_{\mathrm{c}, \mathrm{CH}_{4}}=4.599 \mathrm{MPa}, \omega_{\mathrm{CH}_{4}}=0.011, T_{\mathrm{c}, \mathrm{H}_{2} \mathrm{~S}}=$ $373.4 \mathrm{~K}, p_{\mathrm{c}, \mathrm{H}_{2} \mathrm{~S}}=8.963$ andMPa, $\left.\omega_{\mathrm{H}_{2} \mathrm{~S}}=0.09\right)$ additional critical points at $x_{\mathrm{CH}_{4}}=0.84$ can be found. Furthermore, the binary mixture of $\mathrm{CH}_{4}+\mathrm{H}_{2} \mathrm{~S}$ was not the primary focus of the development of the GERG-2008 equation of state, because these models were primarily designed to describe typical natural gas mixtures. This mixture was chosen in this work for validation of the proposed algorithms; more detailed results for will be discussed in the results section of this paper. 


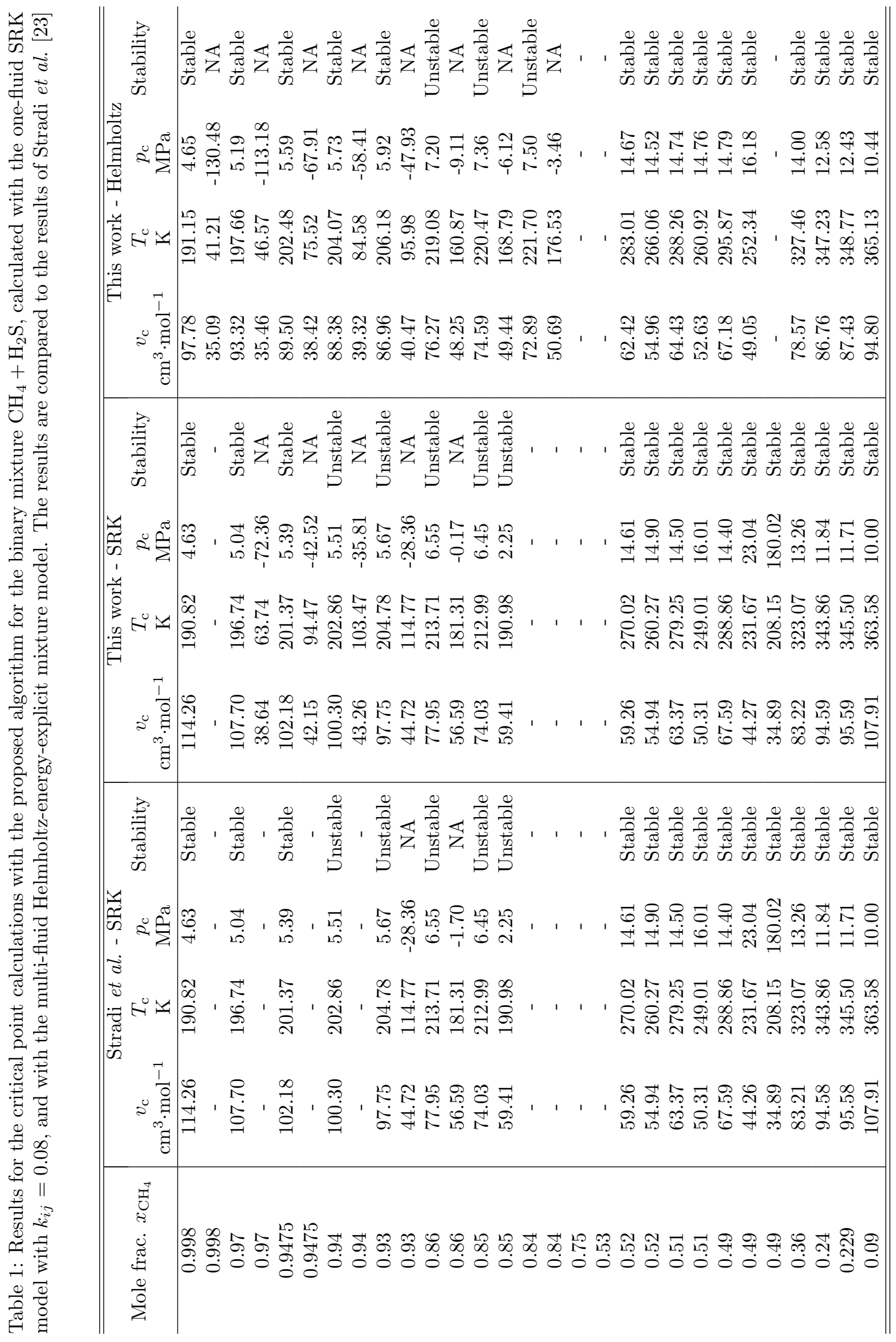




\section{Illustrative results}

There is a practically infinite set of binary mixtures that can be constructed from the fluids for which $T_{c}, p_{c}$, and the acentric factor $\omega$ are known. These binary mixtures can result in wildly varying phase equilibrium behavior. In this section we focus on a few families of fluids that have been studied by other authors in order to validate the methodology, to investigate some details of the algorithm, and further highlight some of the numerical challenges inherent in calculating critical points with multi-fluid models.

Table 2 provides the references for the pure fluids in the multi-fluid model, as well as the pure-component constants used in the cubic equations of state.

\subsection{Methane + hydrogen sulfide}

In this section, the binary mixture of methane and hydrogen sulfide is studied. This binary mixture is often selected for discussions of critical lines, phase diagrams, and related phenomena as it can yield zero, one, two, or even three critical points depending on the molar composition and the equation of state used.

Figure 3 shows the critical points calculated with both the multi-fluid model and the SRK model. The value of $k_{i j}=0.08$ for the SRK model was selected for consistency with the work of Stradi et al. [23], who investigated the same system with the same equation of state (see section 5). With the selected value of $k_{i j}$ for the SRK model, both models yield qualitatively similar results, demonstrating a single critical point up to a methane mole fraction of approximately 0.4 , followed by two critical points up to a mole fraction of approximately 0.52 , no critical points until a methane mole fraction of approximately 0.8 , two critical points around a methane mole fraction of 0.8 , and finally one critical point up to nearly pure methane.

Consideration of the criticality contours can provide some additional insight into the behavior of the critical point solving algorithm and some potential challenges. Figure 4 shows the criticality contours for three different molar compositions, demonstrating the range of behaviors that are possible. In this figure some undesirable behavior of the mixture model can be seen, caused by numerical artifacts of the pure-fluid equations of state. Each of the physical critical points along the $\mathcal{L}_{1}^{*}=0$ contour are highlighted with a marker in the figure, while there are a number of spurious "ghost" critical points that fulfill the mathematical conditions for the existence of a critical point. These "ghost" critical points are all inside the $\mathcal{L}_{1}^{*}=0$ spinodal, and are by definition nonphysical and can be neglected.

In spite of their non-physical nature, the "ghost" critical points cause challenges for the critical point algorithm. These problems are manifested in two primary ways:

1. If the initial guess value for the one-dimensional solver for $\mathcal{L}_{1}^{*}=0$ from section 3.2.1 causes the solver to overshoot and yield a value for $\tau$ inside the spinodal, the presence of values fulfilling $\mathcal{L}_{1}^{*}=0$ inside the spinodal can cause the solver to get stuck. Beginning the solver fairly close to the spinodal removes this potential trap. For instance, Fig. 4b demonstrates that for $\delta=0.5$ and at a value of $\tau$ of approximately 1.32 , the criticality condition $\mathcal{L}_{1}^{*}=0$ is satisfied, though the state is significantly inside the spinodal.

2. While tracing the $\mathcal{L}_{1}^{*}=0$ contour as described in section 3.2.2, it is possible that there may be "ghost" critical points that are very near the spinodal. While the spinodal itself is quite smooth, and can generally be traversed without major challenges, if the search radius contains both the spinodal curve and a fragment of contour of $\mathcal{L}_{1}^{*}=0$, the one-dimensional search for the angle $\theta$ may result in the solver selecting the non-physical fragment as opposed to the desired point along the spinodal.

The difficulties caused by the "ghost" critical points are primarily caused by the pure-fluid equations of state. Figure 1 demonstrates that well-constructed highaccuracy pure-fluid equations of state can still yield reasonable behavior inside the spinodal. As evidence that the "ghost" critical points are caused by the pure-fluid equations of state, Fig. 5 presents the same contours as Fig. 4 except that each pure-fluid equation of state has been replaced with an SRK Helmholtz energy transformation as described in Bell and Jäger [30] (with the purefluid critical points from Table 2). In comparing Figs. 4 and 5 , it can be seen that the cubic equations of state have eliminated the "ghost" critical points, while yielding similar qualitative locations for the critical points. Unfortunately, the use of the SRK equations of state in the multi-fluid model has eliminated the double critical point at a methane mole fraction of 0.49 . The supplemental material includes a number of additional surface plots for this mixture (demonstrating that the majority of the "ghost" critical points are caused by the methane equation of state) and contour plots for the one-fluid SRK model. 
Table 2: Pure-fluid and mixture models and critical values used in this study (EOS: reference for equation of state, BIP: reference for binary interaction parameters in the multi-fluid model)

\begin{tabular}{ccccccccccc}
\hline \hline Fluid 1 & Fluid 2 & EOS 1 & EOS 2 & BIP & $T_{c, 1}(\mathrm{~K})$ & $T_{c, 2}(\mathrm{~K})$ & $p_{\mathrm{c}, 1}(\mathrm{MPa})$ & $p_{\mathrm{c}, 2}(\mathrm{MPa})$ & $\omega_{1}$ & $\omega_{2}$ \\
\hline$n$-Hexane & $n$-Heptane & {$[48]$} & {$[48]$} & {$[25]$} & 507.820 & 540.130 & 3.034 & 2.736 & 0.299 & 0.349 \\
Nitrogen & Methane & {$[49]$} & {$[45]$} & {$[25]$} & 126.192 & 190.564 & 3.396 & 4.599 & 0.037 & 0.011 \\
Methane & $\mathrm{H}_{2} \mathrm{~S}$ & {$[45]$} & {$[46]$} & {$[25]$} & 190.564 & 373.100 & 4.599 & 9.000 & 0.011 & 0.101 \\
Nitrogen & Ethane & {$[49]$} & {$[50]$} & {$[25]$} & 126.192 & 305.322 & 3.396 & 4.872 & 0.037 & 0.099 \\
$\mathrm{CO}_{2}$ & $n$-Propane & {$[51]$} & {$[52]$} & {$[25]$} & 304.128 & 369.890 & 7.377 & 4.251 & 0.224 & 0.152 \\
$\mathrm{CO}_{2}$ & $n$-Pentane & {$[51]$} & {$[48]$} & {$[25]$} & 304.128 & 469.700 & 7.377 & 3.370 & 0.224 & 0.251 \\
$\mathrm{CO}_{2}$ & $n$-Heptane & {$[51]$} & {$[48]$} & {$[25]$} & 304.128 & 540.130 & 7.377 & 2.736 & 0.224 & 0.349 \\
$\mathrm{CO}_{2}$ & $n$-Decane & {$[51]$} & {$[46]$} & {$[25]$} & 304.128 & 617.700 & 7.377 & 2.103 & 0.224 & 0.488 \\
\hline \hline
\end{tabular}
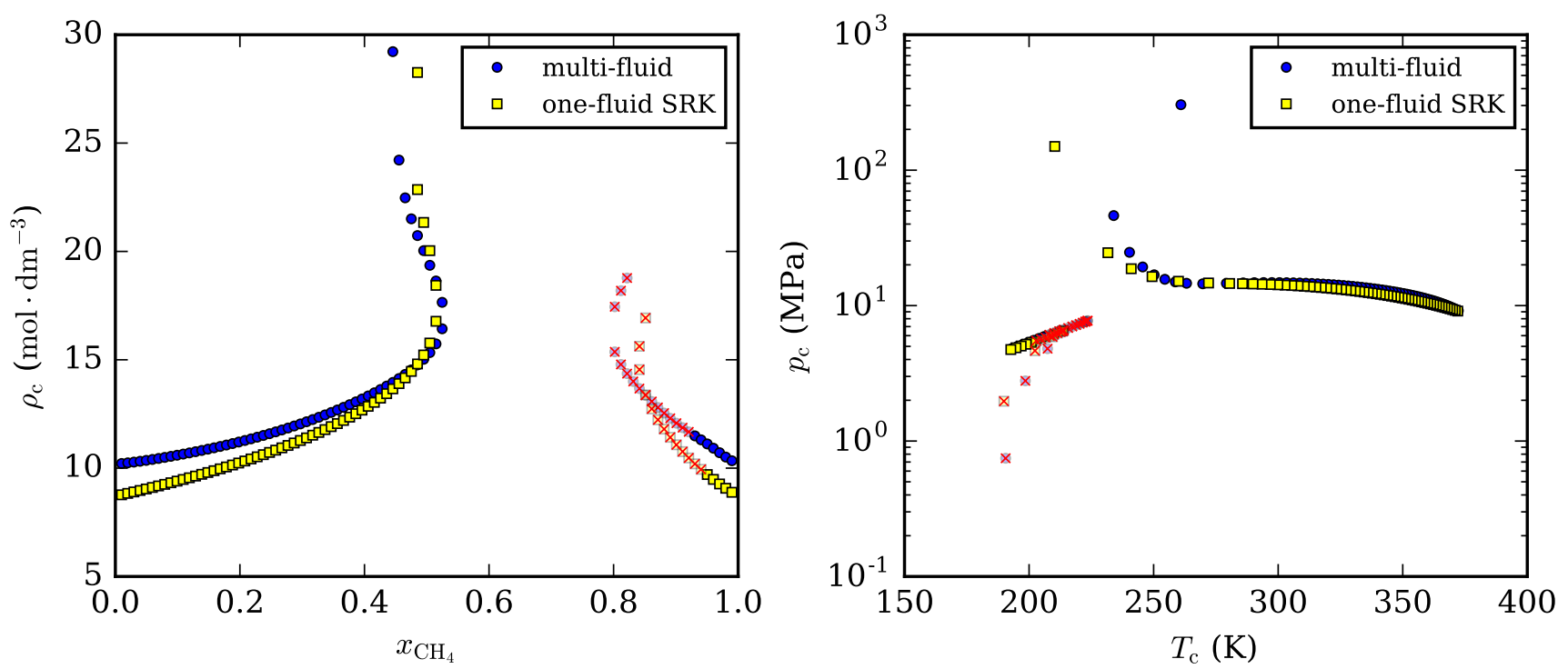

Figure 3: Critical densities, temperatures, and pressures calculated for methane + hydrogen sulfide binary mixtures through the use of the multi-fluid Helmholtz-energy-explicit equation of state and the one-fluid SRK equation of state (crossed-out points are determined to be unstable according to the stability evaluation) 

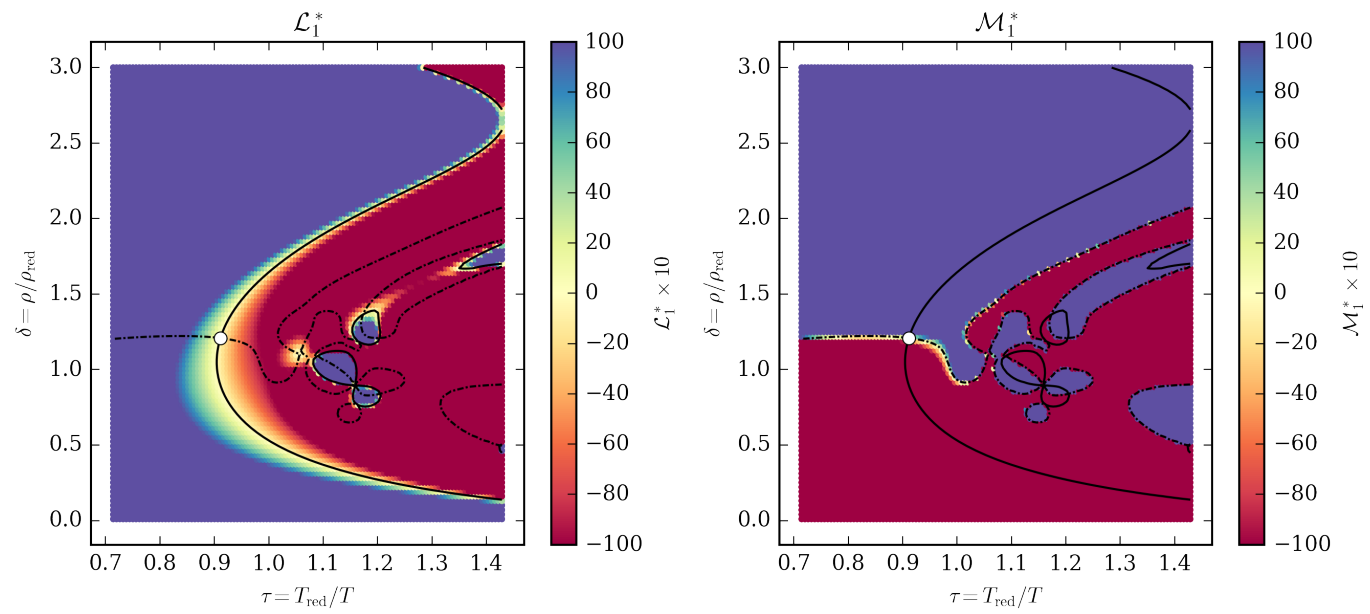

(a) Methane[0.3] \& $\mathrm{H}_{2} \mathrm{~S}[0.7]$ demonstrating one critical point
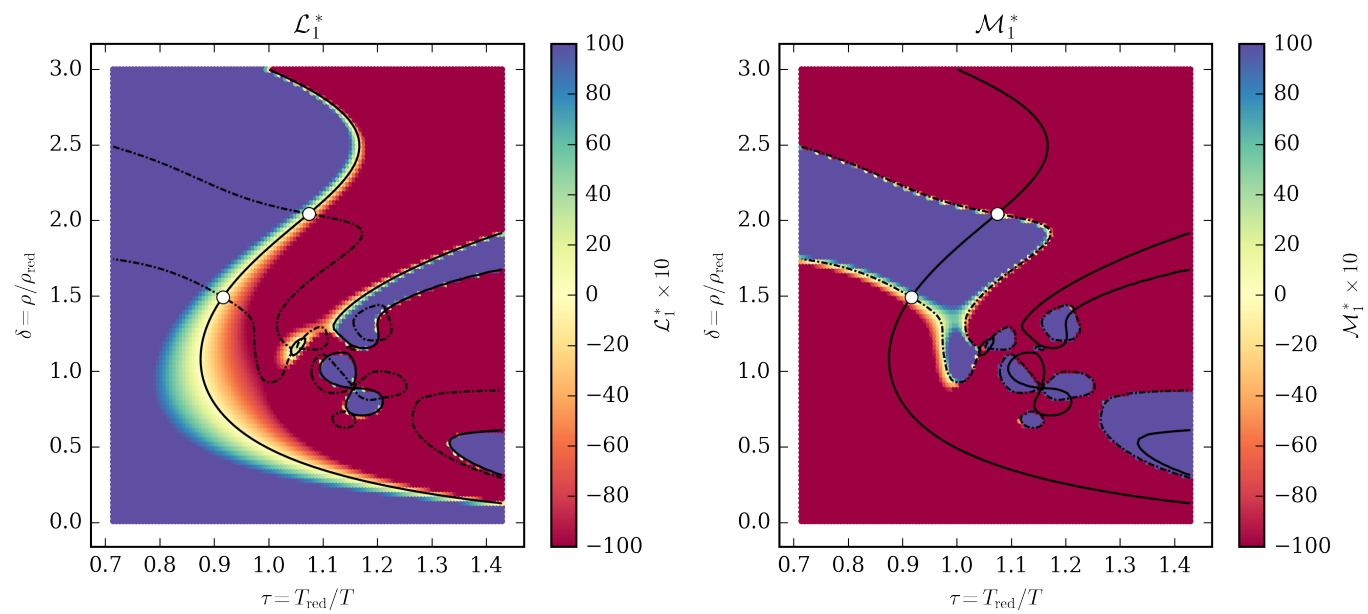

(b) Methane[0.49] \& $\mathrm{H}_{2} \mathrm{~S}[0.51]$ demonstrating two critical points
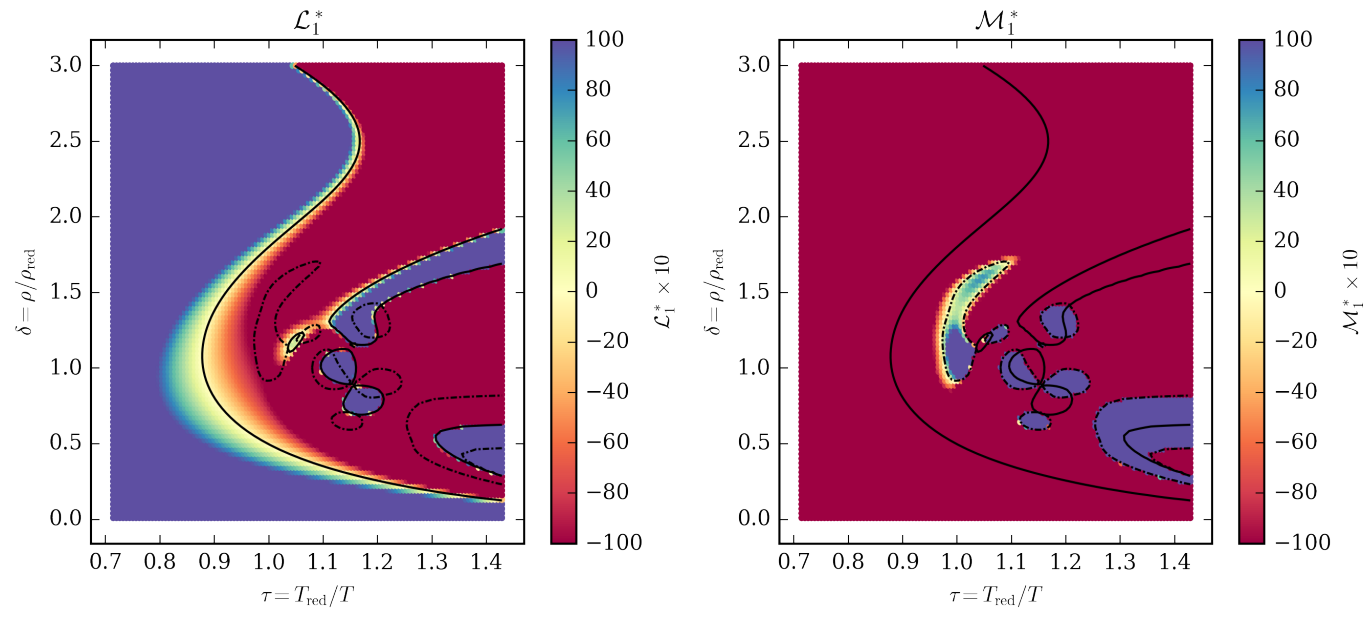

(c) Methane[0.65] \& $\mathrm{H}_{2} \mathrm{~S}[0.35]$ demonstrating zero critical points

Figure 4: Plots of surfaces for $\mathcal{L}_{1}^{*}$ and $\mathcal{M}_{1}^{*}$ for a selection of methane/hydrogen sulfide mixtures (molar composition). The $\mathcal{L}_{1}^{*}=0$ (solid) and $\mathcal{M}_{1}^{*}=0$ (dashed) contours are plotted on each surface. 

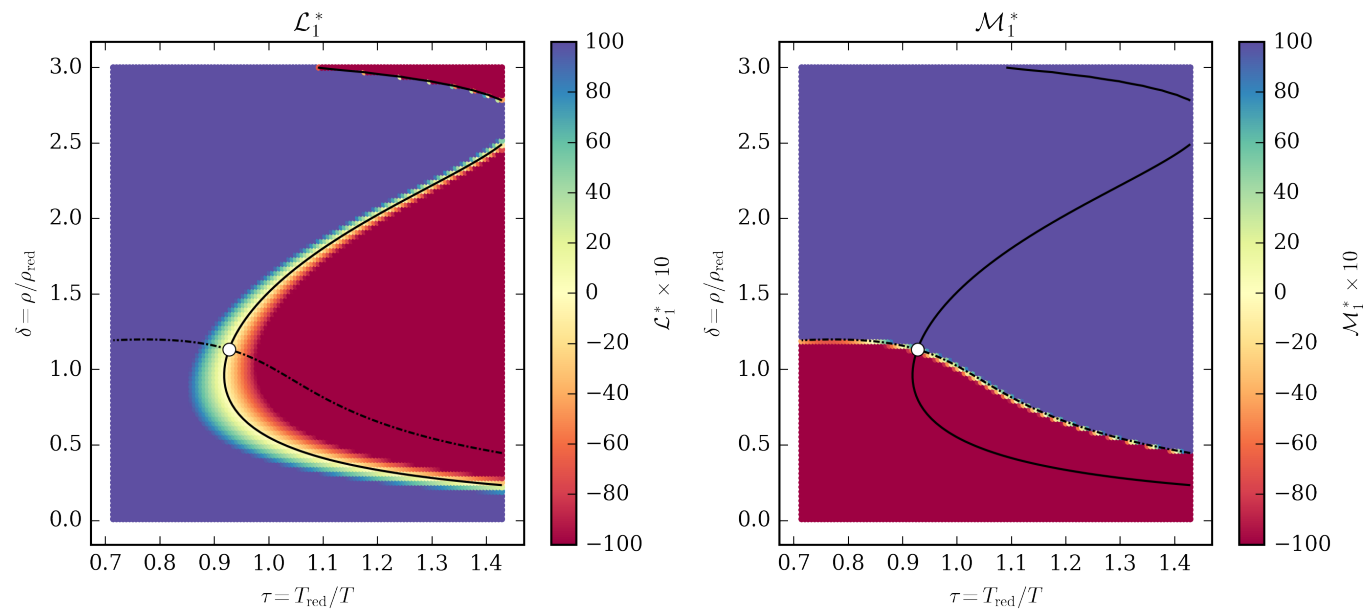

(a) Methane[0.3] \& $\mathrm{H}_{2} \mathrm{~S}[0.7]$ demonstrating one critical point
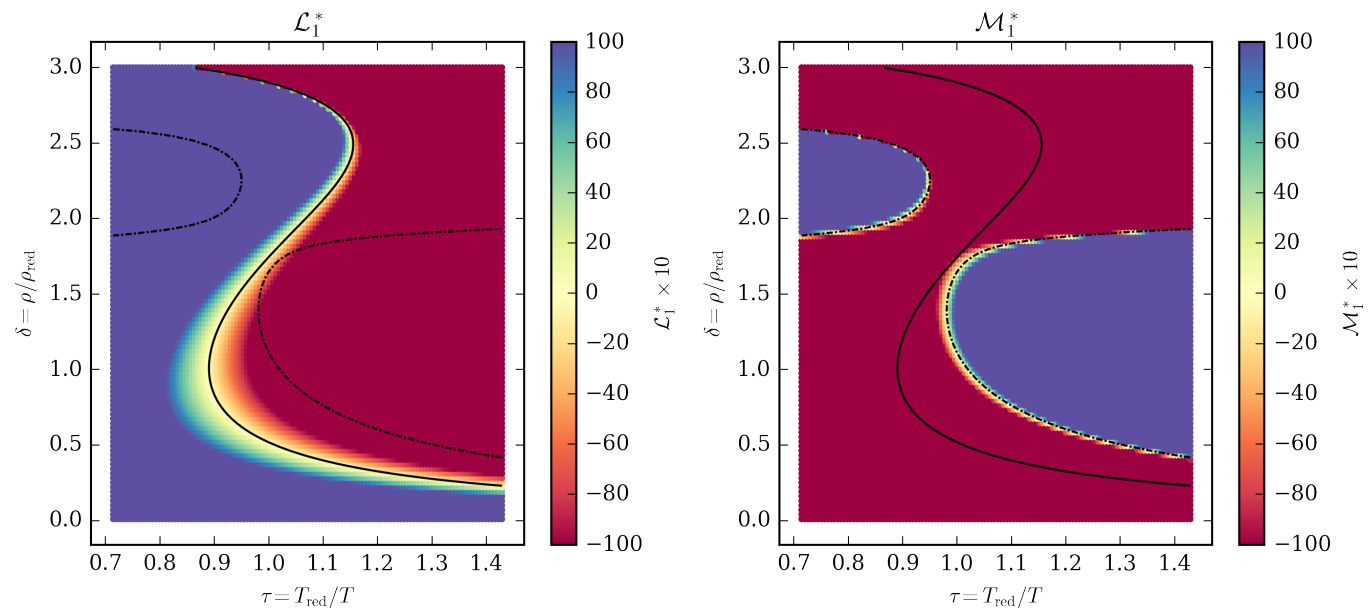

(b) Methane[0.49] \& $\mathrm{H}_{2} \mathrm{~S}[0.51]$ demonstrating zero critical points
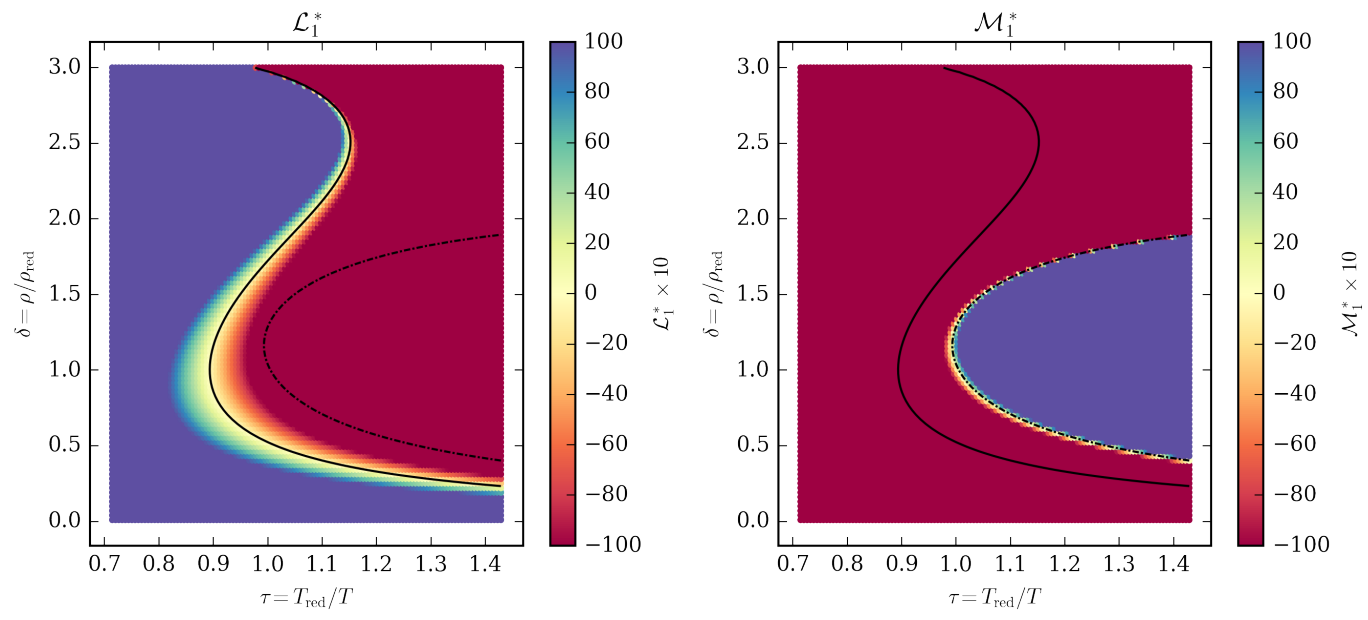

(c) Methane[0.65] \& $\mathrm{H}_{2} \mathrm{~S}[0.35]$ demonstrating zero critical points

Figure 5: Plots of surfaces for $\mathcal{L}_{1}^{*}$ and $\mathcal{M}_{1}^{*}$ for a methane $+\mathrm{H}_{2} \mathrm{~S}$ mixture when both fluids in the multi-fluid model are replaced with SRK transformations. See Fig. 4 for a description of the content. 


\subsection{Nitrogen + ethane}

Figure 6 presents the critical points for the binary mixture of nitrogen and ethane. The value of the one-fluid SRK mixture model interaction parameter $k_{i j}=0.0407$ is taken from Ramírez-Jiménez et al. [53]. This mixture is of interest because even though the constituent fluids are simple molecules, and form a relatively symmetric mixture, it is still possible to yield interesting critical point behavior. Over a range of nitrogen mole fractions between approximately 0.6 and 0.7 , as many as three stable critical points can be found.

Figure 7 shows the criticality contours for a nitrogen + ethane mixture at a nitrogen mole fraction of 0.65 . There are three crossings of the critical contours along the main branch of the $\mathcal{L}_{1}^{*}=0$ contour, demonstrating that three critical points are predicted. All three of the critical points are predicted to be stable, but the critical point finder does not find the highest pressure critical point because it is located at a pressure greater than 500 $\mathrm{MPa}$, one of the stopping criteria for the spinodal tracer.

\subsection{Carbon dioxide + linear alkanes}

Another popular family of binary mixtures of technical relevance are the mixtures of carbon dioxide and the linear alkanes. These mixtures tend to be of Type I, with a continuous critical line between the critical points of the pure fluids, at least for the lower alkanes below a carbon number of approximately 10 or 11. For higher alkanes (for instance hexadecane), non-Type I phase behavior is seen for mixtures with carbon dioxide.

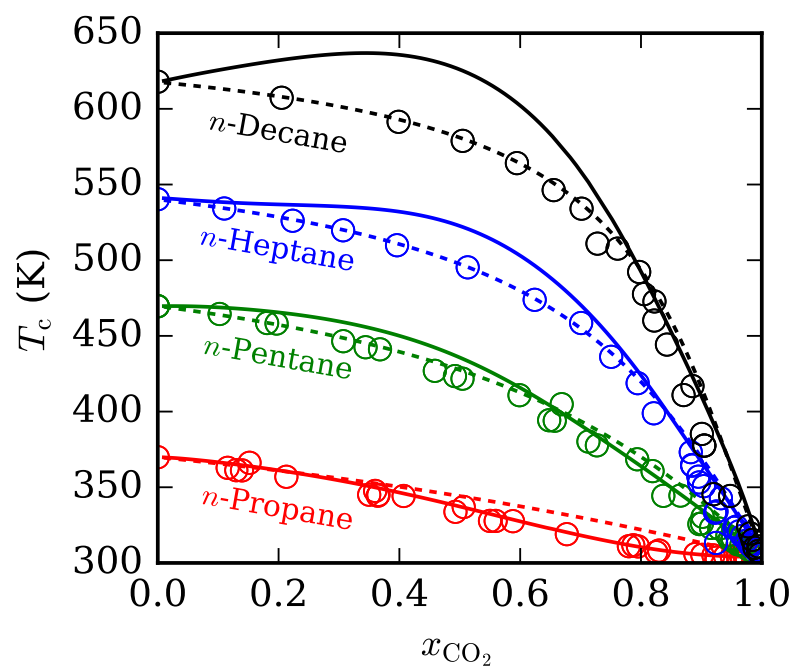

Figure 8: Critical temperature of binary carbon dioxide $+n$-alkane mixtures as in the work of Kolár [10] (solid: multi-fluid model, dashes: SRK as one-fluid model with $k_{i j}=0$, markers: experimental measurements from the TDE database $[54,55,56])$, with parameters taken from Table 2

Figure 8 shows the critical lines calculated with the multi-fluid model and the one-fluid SRK model with $k_{i j}=0$. For the higher alkanes, the predictions of the critical temperatures are excellent and the SRK model captures the shape of the critical line with high accuracy. Conversely, for the higher alkanes the multi-fluid model provides predictions of the critical temperatures that are significantly too high. This deviating behavior is a manifestation of the fact that when the binary interaction parameters were fit in the work of Kunz \& Wagner [25], the critical points were not considered and therefore the binary interaction parameters were not constrained to yield the correct critical lines. Furthermore, the GERG model was not primarily designed for carbon dioxide rich mixtures. In general, as the mixture becomes more asymmetric, the deviations from the multifluid model increase.

For propane, the conclusions are exactly opposite those for the higher alkanes. The highest-accuracy predictions of the critical temperatures can be obtained from the multi-fluid model, and the one-fluid SRK model deviates more strongly from the experimental data. This is likely just a fortuitous behavior, as the critical points were not considered in the fitting of Kunz \& Wagner [25].

\section{Conclusions}

In this work we have combined the methods of Heideman and Khalil [7] and Hicks and Young [17], and applied 

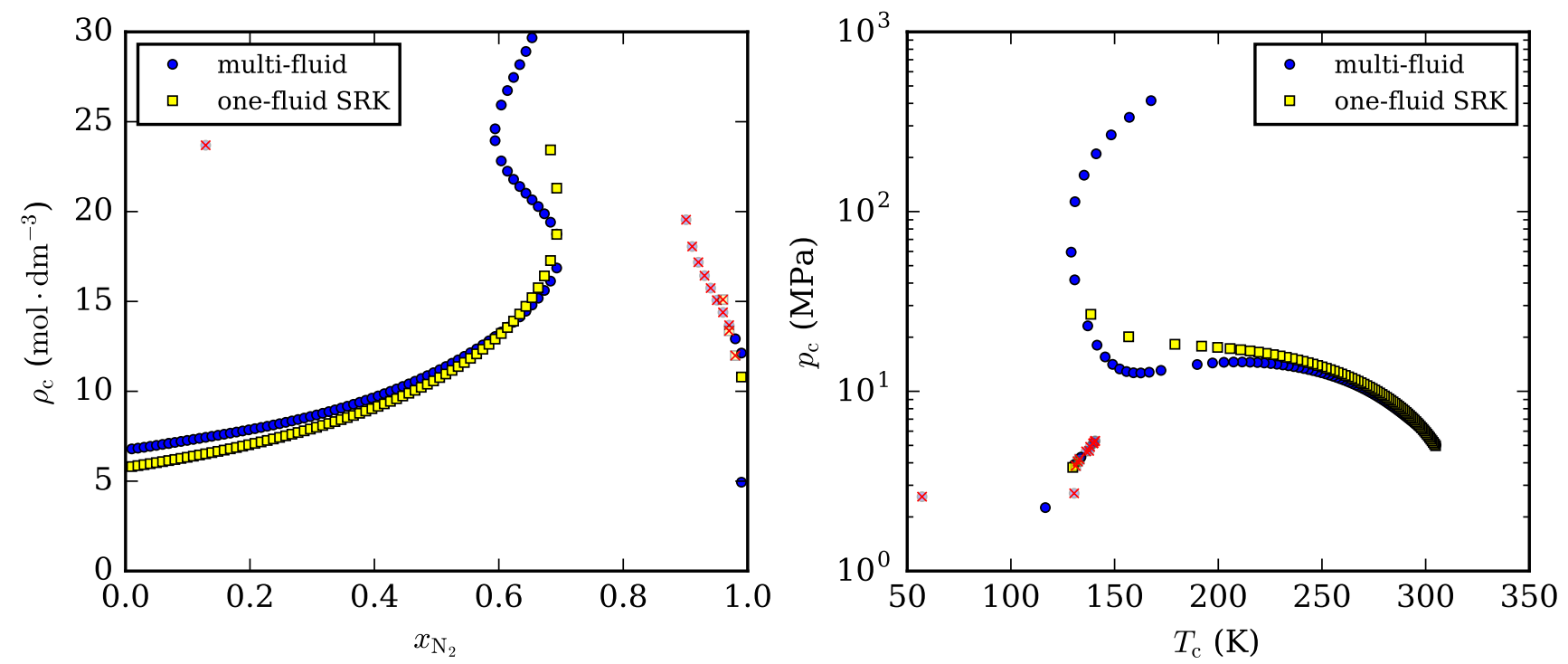

Figure 6: Critical densities, temperatures, and pressures calculated for nitrogen + ethane binary mixtures through the use of the multi-fluid Helmholtz-energy-explicit equation of state and the one-fluid SRK equation of state (crossed-out points are determined to be unstable according to the stability evaluation)
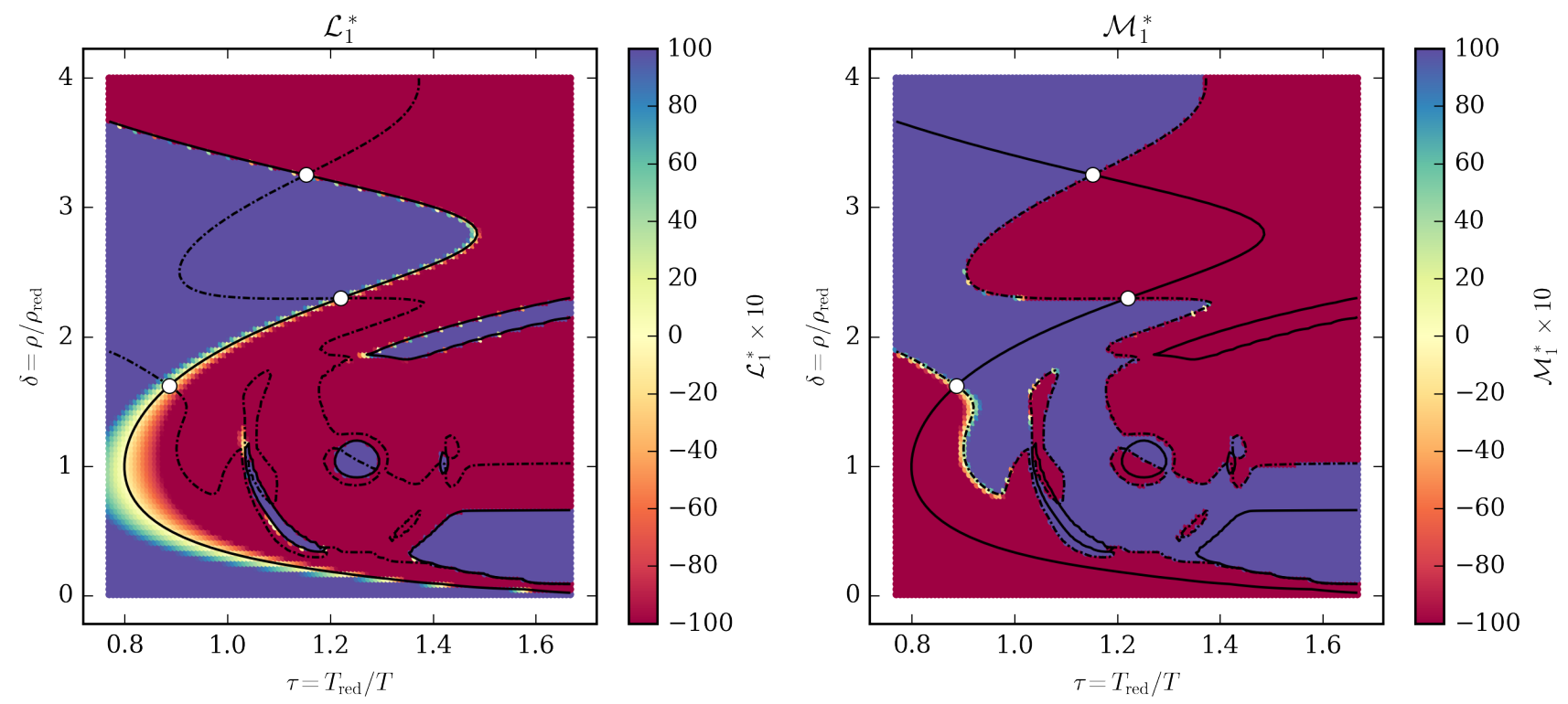

Figure 7: Plots of surfaces for $\mathcal{L}_{1}^{*}$ and $\mathcal{M}_{1}^{*}$ for a nitrogen[0.65] + ethane[0.35] mixture demonstrating three critical points when using the multi-fluid mixture model. See Fig. 4 for a description of the content. 
them to multi-fluid Helmholtz-energy-explicit mixture models. This new algorithm allows for the robust determination of the critical points of complex mixtures. It is demonstrated that unphysical behavior of the pure fluid equations of state in the multi-fluid mixture model is of great importance, and poorly behaving pure-fluid equations of state can cause significant challenges in terms of locating the critical points.

\section{Acknowledgments}

The authors thank Eric Lemmon (of NIST), who provided daily inspiration, G. Venkatarathnam (of IIT Madras), who helped with the literature survey, Bradley Alpert (of NIST), who provided invaluable assistance with the linear algebra analysis, Andrei Kazakov (of NIST), who suggested the form of the fit for $v_{\mathrm{c}, i}$, and Arno Laesecke (of NIST), who brought to our attention the discussions in literature of the proper use of the term "excess".

\section{References}

[1] A. Jäger. Complex Phase Equilibria of Gas Hydrates and other Solid and Fluid Phases Modeled With Highly Accurate Equations of State. PhD thesis, Ruhr-Universität Bochum, 2015.

[2] J. W. Gibbs. The Scientific Papers of J. Willard Gibbs, Ph.D. LL.D., chapter On The Equilibrium of Heterogeneous Substances, pages 55-353. Longmans, Green, and Co., 1906.

[3] B. L. Beegle, M. Modell, and R. C. Reid. Thermodynamic Stability Criterion for Pure Substance and Mixtures. AIChE J., 20(4):1200-1206, 1974. doi: 10.1002/aic.690200621.

[4] C. Hicks and C. Young. Gas-liquid critical properties of binary mixtures. Chem. Rev., 1975. doi: $10.1021 /$ cr60294a001.
[5] R. C. Reid and B. L. Beegle. Critical Point Criteria in Legendre Transform Notation. AIChE J., 23(5):726-732, 1977. doi: 10.1002/aic.690230515.

[6] K. Gubbins and C. Twu. Thermodynamics of Polyatomic Fluid Mixtures - I: Theory. Chem. Eng. Sci., 33:863-878, 1978. doi: 10.1016/00092509(78)85176-8.

[7] R. A. Heidemann and A. M. Khalil. The Calculation of Critical Points. AIChE J., 26(5):769-778, 1980. doi: 10.1002/aic.690260510.

[8] M. L. Michelsen. Calculation of critical points and phase boundaries in the critical region. Fluid Phase Equilib., 16:57-76, 1984. doi: 10.1016/03783812(84)85021-9.

[9] R. J. Sadus. Calculating critical transitions of fluid mixtures: Theory vs. experiment. AIChE J., 40(8):1376-1403, 1994. doi: 10.1002/aic.690400810.

[10] P. Kolář and K. Kojima. Prediction of critical points in multicomponent systems using the PSRK group contribution equation of state. Fluid Phase Equilib., 118(2):175-200, 1996. doi: 10.1016/03783812(95)02850-1.

[11] M. L. Michelsen and J. M. Mollerup. Thermodynamic Models: Fundamentals \& Computational Aspects. Tie-Line Publications, 2007.

[12] U. K. Deiters and T. Kraska. High-Pressure Fluid Phase Equilibria: Phenomenology and Computation. Elsevier, 2012.

[13] P. H. V. Konynenburg and R. L. Scott. Critical Lines and Phase Equilibria in Binary Van Der Waals Mixtures. Philosophical Transactions of the Royal Society of London. Series A, Mathematical and Physical Sciences, 1980. doi: $10.1098 /$ rsta. 1980.0266 .

[14] R. L. Scott and P. H. van Konynenburg. Van der Waals and Related Models for Hydrocarbon Mixtures. Discussions of the Faraday Society, 1970. doi: 10.1039/DF9704900087.

[15] U. K. Deiters and I. L. Pegg. Systematic investigation of the phase behavior in binary fluid mixtures. i. calculations based on the redlichkwong equation of state. The Journal of Chemical Physics, 90(11):6632-6641, 1989. doi: http://dx.doi.org/10.1063/1.456280.

[16] T. Kraska and U. K. Deiters. Systematic investigation of the phase behavior in binary fluid mixtures. ii. calculations based on the carnahanstarlingredlichkwong equation of state. The Journal 
of Chemical Physics, 96(1):539-547, 1992. doi: http://dx.doi.org/10.1063/1.462490.

[17] C. P. Hicks and C. L. Young. Theoretical prediction of phase behaviour at high temperatures and pressures for non-polar mixtures. Part 1.-Computer solution techniques and stability tests. J. Chem. Soc., Faraday Trans. 2, 73:597-612, 1977. doi: 10.1039/F29777300597.

[18] M. Cismondi and M. L. Michelsen. Global phase equilibrium calculations: Critical lines, critical end points and liquid-liquid-vapour equilibrium in binary mixtures. J. Supercrit. Fluids, 39(3):287-295, 2007. doi: 10.1016/j.supflu.2006.03.011.

[19] K. S. Patel. Automatic generation of global phase equilibrium diagram from equation of state. $\mathrm{PhD}$ thesis, University of South Florida, 2007.

[20] K. S. Patel and A. K. Sunol. Automatic generation of global phase equilibrium diagrams for binary systems from equations of state. Comput. Chem. Eng., 33(11):1793-1804, 2009. doi: 10.1016/j.compchemeng.2009.03.004.

[21] A. van Pelt, C. Peters, and J. de Swaan Arons. Calculation of critical lines in binary mixtures with the simplified perturbed hard chain theory. Fluid Phase Equilib., 84:23-47, 1993. doi: 10.1016/03783812(93)85116-4.

[22] H. Hoteit, E. Santiso, and A. Firoozabadi. An efficient and robust algorithm for the calculation of gas-liquid critical point of multicomponent petroleum fluids. Fluid Phase Equilib., 2006. doi: 10.1016/j.fluid.2005.12.019.

[23] B. A. Stradi, J. F. Brennecke, P. Kohn, and M. A. Stadtherr. Reliable computation of mixture critical points. AIChE J., 2001. doi: 10.1002/aic.690470121.

[24] O. Kunz, R. Klimeck, W. Wagner, and M. Jaeschke. The GERG-2004 Wide-Range Equation of State for Natural Gases and Other Mixtures. VDI Verlag GmbH, 2007.

[25] O. Kunz and W. Wagner. The GERG-2008 WideRange Equation of State for Natural Gases and Other Mixtures: An Expansion of GERG-2004. J. Chem. Eng. Data, 57:3032-3091, 2012. doi: 10.1021/je300655b.

[26] E. W. Lemmon. A Generalized Model for the Prediction of the Thermodynamic Properties of Mixtures Including Vapor-Liquid Equilibrium. PhD thesis, University of Idaho, Moscow, 1996.
[27] E. W. Lemmon and R. Tillner-Roth. A Helmholtz energy equation of state for calculating the thermodynamic properties of fluid mixtures. Fluid Phase Equilib., 165:1-21, 1999.

[28] E. W. Lemmon and R. T. Jacobsen. A Generalized Model for the Thermodynamic Properties of Mixtures. Int. J. Thermophys., 20(3):825-835, 1999. doi: $10.1023 / \mathrm{A}: 1022627001338$.

[29] K. N. Marsh. Editorial. J. Chem. Eng. Data, 42(1):1, 1997. doi: 10.1021/je9604996.

[30] I. H. Bell and A. Jäger. Helmholtz energy transformations of common cubic equations of state for use with pure fluids and mixtures. J. Res. NIST, 2016.

[31] D.-Y. Peng and D. B. Robinson. A new two-constant equation of state. Ind. Eng. Chem. Fundamen., 15(1):59-64, $1976 . \quad$ doi: 10.1021/i160057a011.

[32] D. B. Robinson and D.-Y. Peng. The characterization of the heptanes and heavier fractions for the GPA Peng-Robinson programs (Research Report RR-28). Gas Processors Association, 1978.

[33] G. Soave. Equilibrium Constants from a Modified Redlich-Kwong Equation of State. Chem. Eng. Sci., 27:1197-1203, 1972. doi: 10.1016/00092509(72)80096-4.

[34] R. Akasaka. Calculation of the critical point for mixtures using mixture models based on Helmholtz energy equations of state. Fluid Phase Equilib., 263:102-108, 2008. doi: 10.1016/j.fluid.2007.10.007.

[35] E. Lemmon, M. Huber, and M. McLinden. NIST Standard Reference Database 23: Reference Fluid Thermodynamic and Transport PropertiesREFPROP, Version 9.0, 2010.

[36] I. H. Bell, J. Wronski, S. Quoilin, and V. Lemort. Pure and Pseudo-pure Fluid Thermophysical Property Evaluation and the Open-Source Thermophysical Property Library CoolProp. Ind. Eng. Chem. Res., 53(6):2498-2508, 2014. doi: 10.1021/ie4033999.

[37] R. Span, T. Eckermann, S. Herrig, S. Hielscher, A. Jäger, and M. Thol. TREND. Thermodynamic Reference and Engineering Data 2.0, 2015.

[38] J. Gernert, A. Jäger, and R. Span. Calculation of phase equilibria for multi-component mixtures using highly accurate Helmholtz energy equations of state. Fluid Phase Equilib., 375:209-218, 2014. doi: 10.1016/j.fluid.2014.05.012. 
[39] J. Gernert and R. Span. EOS-CG: A Helmholtz energy mixture model for humid gases and CCS mixtures. J. Chem. Thermodyn., 93:274-293, 2016. doi: 10.1016/j.jct.2015.05.015.

[40] J. R. Magnus and H. Neudecker. Matrix Differential Calculus with Applications in Statistics and Econometrics, Third Edition. John Wiley and Sons, 2007.

[41] M. Michelsen. The isothermal flash problem. Part I. Stability. Fluid Phase Equilib., 9:1-19, 1982. doi: 10.1016/0378-3812(82)85001-2.

[42] M. Michelsen. The isothermal flash problem. Part II. Phase-split calculation. Fluid Phase Equilib., 9:21-40, 1982. doi: 10.1016/0378-3812(82)85002-4.

[43] A. Péneloux, E. Rauzy, and R. Fréze. A Consistent Correction for Redlich-Kwong-Soave Volumes. Fluid Phase Equilib., 8(1):7-23, 1982. doi: 10.1016/0378-3812(82)80002-2.

[44] R. C. Reid, J. M. Prausnitz, and B. E. Poling. The properties of gases and liquids. McGraw-Hill, 1987.

[45] U. Setzmann and W. Wagner. A New Equation of State and Tables of Thermodynamic Properties for Methane Covering the Range from the Melting Line to $625 \mathrm{~K}$ at Pressures up to $1000 \mathrm{MPa}$. J. Phys. Chem. Ref. Data, 20(6):1061-1151, 1991. doi: 10.1063/1.555898.

[46] E. Lemmon and R. Span. Short Fundamental Equations of State for 20 Industrial Fluids. J. Chem. Eng. Data, 51:785-850, 2006. doi: 10.1021/je050186n.

[47] B. E. Poling, J. M. Prausnitz, and J. P. O'Connell. The Properties of Gases and Liquids, 5th edition. McGraw Hill, 2001.

[48] R. Span and W. Wagner. Equations of State for Technical Applications. II. Results for Nonpolar Fluids. Int. J. Thermophys., 24:41-109, 2003. doi: 10.1023/A:1022310214958.

[49] R. Span, E. W. Lemmon, R. T. Jacobsen, W. Wagner, and A. Yokozeki. A Reference Equation of State for the Thermodynamic Properties of Nitrogen for Temperatures from 63.151 to $1000 \mathrm{~K}$ and Pressures to 2200 MPa. J. Phys. Chem. Ref. Data, 29:13611433, 2000. doi: 10.1063/1.1349047.
[50] D. Buecker and W. Wagner. A Reference Equation of State for the Thermodynamic Properties of Ethane for Temperatures from the Melting Line to $675 \mathrm{~K}$ and Pressures up to $900 \mathrm{MPa}$. J. Phys. Chem. Ref. Data, 35(1):205-266, 2006. doi: 10.1063/1.1859286.

[51] R. Span and W. Wagner. A New Equation of State for Carbon Dioxide Covering the Fluid Region from the Triple Point Temperature to $1100 \mathrm{~K}$ at Pressures up to $800 \mathrm{MPa}$. J. Phys. Chem. Ref. Data, 25:1509-1596, 1996. doi: 10.1063/1.555991.

[52] E. W. Lemmon, M. O. McLinden, and W. Wagner. Thermodynamic Properties of Propane. III. A Reference Equation of State for Temperatures from the Melting Line to $650 \mathrm{~K}$ and Pressures up to 1000 MPa. J. Chem. Eng. Data, 54:3141-3180, 2009. doi: $10.1021 /$ je900217v

[53] E. Ramírez-Jiménez, D. N. Justo-García, F. GarcíaSánchez, and R. P. Stateva. VLL Equilibria and Critical End Points Calculation of Nitrogen-Containing LNG Systems: Application of SRK and PC-SAFT Equations of State. Ind. Eng. Chem. Res., 51(27):9409-9418, 2012. doi: 10.1021/ie300372a.

[54] V. Diky, R. D. Chirico, C. D. Muzny, A. F. Kazakov, K. Kroenlein, J. W. Magee, I. Abdulagatov, J. W. Kang, and M. Frenkel. ThermoData Engine (TDE) software implementation of the dynamic data evaluation concept. 7 . Ternary mixtures. J. Chem. Inf. Model., 52(1):260-276, 2011. doi: 10.1021/ci200456w.

[55] V. Diky, R. D. Chirico, C. D. Muzny, A. F. Kazakov, K. Kroenlein, J. W. Magee, I. Abdulagatov, J. W. Kang, R. Gani, and M. Frenkel. ThermoData Engine (TDE): Software implementation of the dynamic data evaluation concept. 8 . Properties of material streams and solvent design. J. Chem. Inf. Model., 53(1):249-266, 2012. doi: 10.1021/ci300470t.

[56] V. Diky, R. D. Chirico, C. D. Muzny, A. F. Kazakov, K. Kroenlein, J. W. Magee, I. Abdulagatov, and M. Frenkel. ThermoData Engine (TDE): Software implementation of the dynamic data evaluation concept. 9. Extensible thermodynamic constraints for pure compounds and new model developments. $J$. Chem. Inf. Model., 53(12):3418-3430, 2013. doi: 10.1021/ci4005699. 